\title{
MWCNT-Coated Cotton Yarn Array For Piezoresistive Force and Bending Sensor Applications in Internet of Things Systems
}

Dae-Young Jeon ( $\boldsymbol{\nabla}$ dyjeon@kist.re.kr)

Korea Institute of Science and Technology

So Jeong Park

Korea University

Tae Yoon Lee

Korea Institute of Science and Technology

Gyu-Tae Kim

Korea University

\section{Research Article}

Keywords: Multi-walled carbon nanotube (MWCNT), MWCNT-coated cotton yarn, force sensor, bending sensor, sensor matrix, real-time data storage in cloud system, open-source microcontroller, Internet of Things (loT)

Posted Date: February 24th, 2021

DOl: https://doi.org/10.21203/rs.3.rs-225786/v1

License: (c) (1) This work is licensed under a Creative Commons Attribution 4.0 International License. Read Full License

Version of Record: A version of this preprint was published at Sensors and Actuators A: Physical on November 1st, 2021. See the published version at https://doi.org/10.1016/j.sna.2021.113209. 


\title{
MWCNT-coated cotton yarn array for piezoresistive force and bending sensor applications in Internet of Things systems
}

\author{
Dae-Young Jeon ${ }^{1, a)}$, So Jeong Park ${ }^{2}$, Tae Yoon Lee ${ }^{1}$, Gyu-Tae Kim² \\ ${ }^{1}$ Institute of Advanced Composite Materials, Korea Institute of Science and Technology, \\ Joellabuk-do 55324, Korea \\ ${ }^{2}$ School of Electrical Engineering, Korea University, Seoul 136-701, Korea
}

${ }^{a)}$ Corresponding author: dyjeon@kist.re.kr (D.-Y. Jeon)

\begin{abstract}
Advanced sensors based on the Internet of Things (IoT) are bringing new technology and productivity paradigms to the era of the fourth industrial revolution. Here, piezoresistive force and bending sensors with good performance (sensitivity $\approx 0.3 \mathrm{kPa}^{-1}$ and $\Delta$ normalized resistance/ $\Delta$ curvature $\approx 1.5 \mathrm{~cm}$ ) were fabricated by using perpendicularly aligned multi-walled (MW) carbon nanotube-coated yarn (CNT-CY) arrays, which were obtained from a simple dipping-and-drying method. Verification was performed using a proof-of-concept force sensor matrix with an Arduino open-source microcontroller. In addition, a force sensor combined with real-time data storage in Google Cloud was successfully demonstrated. This work provides important information for the development of a robust, low-cost, and easy-to-manufacture IoT platform with MWCNT-based composites as an active sensing material.
\end{abstract}


Keywords: Multi-walled carbon nanotube (MWCNT), MWCNT-coated cotton yarn, force sensor, bending sensor, sensor matrix, real-time data storage in cloud system, open-source microcontroller, Internet of Things (IoT)

\section{Introduction}

Advanced sensing platforms, which can detect, monitor, and predict external environments in real time, have recently attracted considerable attention in the era of the fourth industrial revolution, comprising the Internet of Things (IoT), big data, cloud computing, robotics, and artificial intelligence. ${ }^{[1-5]}$ Advanced sensors based on wireless technologies and Internet growth have facilitated interconnections among things and can be employed in various contexts such as smart factories, smart cities, autonomous vehicles, healthcare, and security systems. ${ }^{[6-8]}$ In addition, open-source hardware (e.g., Arduino, Raspberry Pi, Latte panda, Galileo, and Jetson Nano) enables successful development of low-cost IoT application systems because anyone can freely use, study, modify, produce, and distribute this hardware without licenses and copyright royalties. ${ }^{[9-11]}$ Robust and low-cost materials as active sensing elements of electronic sensors are also important for costeffective and reliable IoT systems. Carbon nanotubes (CNTs) possess extraordinary electrical, mechanical, thermal, and chemical properties with the potential for low-cost mass production. ${ }^{[12]}$ Because of these advantages, CNTs have been used in many outstanding applications, including diodes, transistors, electronic interconnects, thermal interfaces, and energy storage devices. ${ }^{[13]}$ CNT-based high-performance sensors have also been constructed for human motion detection, healthcare monitoring, and wearable electronics. ${ }^{[14,15]}$ In particular, strain and pressure sensors with high sensitivity, light weight, excellent flexibility, and stretchability have been demonstrated using CNT-coated conductive cotton composites without the need for micro-fabrication processes 
or extensive chemical treatments. ${ }^{[16-19]}$ Moreover, CNT-coated cotton thread has great potential in wearable sensors because it can be easily fabricated, embedded, or merged into clothes. ${ }^{[20]}$ In the present work, multi-walled (MW) CNT-coated yarn (CNT-CY) was generated by using a simple dipping-and-drying method, and aligned MWCNT-CY arrays were fabricated on polyethylene terephthalate (PET) or glass substrate. Orientation-dependent impedance characteristics and piezoresistive properties of the MWCNT-CY arrays were investigated. In addition, bending sensors with good sensitivity, force sensors combined with real-time data storage in Google Cloud, and force sensor matrices were successfully demonstrated by using the perpendicularly aligned MWCNT-CY array with an Arduino open-source microcontroller.

\section{Results and discussion}

MWCNT-CY composite was prepared by a simple dipping-and-drying method, as shown in Fig. 1(a). Cotton yarn easily adsorbed the water-based MWCNT solution because of its hierarchical and porous structures. ${ }^{[16,17]}$ Intrinsically insulated cotton yarn was uniformly coated with conductive MWCNTs, and its color was noticeably altered from white and black after the coating process. For further uniform MWCNT-coating, thermal treatment in hot water and ultrasonication can be performed to eliminate a number of impurities on commercial cotton yarns. ${ }^{[16]}$ Figure 1(b) presents the Raman spectrum of MWCNT-CY. The two clear peaks constitute typical behavior of MWCNT. ${ }^{[21,22]}$ The first peak (D-band) at $\approx 1330 \mathrm{~cm}^{-1}$ is related to disorder, structural imperfections of graphitic domains, or impurities. The $\mathrm{E}_{2 \mathrm{~g}}$ vibration mode of the graphene unit cell in the hexagonal crystalline is associated with the second peak at $\approx 1560 \mathrm{~cm}^{-1}$ (G-band). 
MWCNT-CYs were placed and aligned via double-sided adhesive tape on PET substrate. There were two distinct device structures for electrical measurement involving perpendicular and horizontal MWCNT-CY alignment relative to the electrodes, as shown in Fig. 2.

Impedance characterization was performed on the fabricated MWCNT-CY array devices with different orientations. In-depth electrical properties of various materials and their interfaces can be investigated in detail by impedance measurement. ${ }^{[23-25]}$ Total impedance $(Z)$ and phase angle $(\theta$; phase difference between voltage and current waveform) for perpendicularly aligned MWCNTCY arrays are plotted in Fig. 3(a). For a relatively low frequency, ranging from $10 \mathrm{~Hz}$ to $10^{5} \mathrm{~Hz}$, a constant $\mathrm{Z} \approx 12.6 \mathrm{k} \Omega$ and $\theta \approx 0$ degrees were obtained. These results denote a frequencyindependent resistive dominant response. Furthermore, dramatically reduced $\mathrm{Z}$ (up to $\approx 6 \mathrm{k} \Omega$ ) and enhanced $\theta$ (towards 45 degrees) were observed with greater frequency $\left(>10^{5} \mathrm{~Hz}\right)$, indicating a considerable capacitive component (C) and resistance (R). These results are consistent with the typical impedance behavior of an RC parallel equivalent circuit model. ${ }^{[23,24]}$ Individual MWCNTs contribute mainly to the value of $\mathrm{R}$, and the $\mathrm{C}$ component could originate from MWCNTCY/Air/MWCNT-CY junctions. ${ }^{[26,27]}$ For horizontally aligned MWCNT-CY arrays in Fig. 3(b), the $\mathrm{Z}$ and $\theta$ remained constant $(\mathrm{Z} \approx 490 \Omega$ and $\theta \approx 0$ ) for the overall frequency range, and only slight changes in the values were observed at very high frequency (near $10^{7} \mathrm{~Hz}$ ). These results indicate that horizontally aligned MWCNT-CY arrays provide electrical resistance with negligible capacitance. In addition, the sheet resistance $\left(\mathrm{R}_{\mathrm{s}}=\mathrm{R} \times\right.$ width/length $\left.\approx 10.9 \mathrm{k} \Omega / \square\right)$ of perpendicularly aligned MWCNT-CY arrays is much greater than that of horizontally aligned MWCNT-CY arrays $\left(\mathrm{R}_{\mathrm{s}} \approx 567 \Omega / \square\right) .{ }^{[28]}$ 
MWCNT-CY array devices on flexible PET substrate were easily bent, as shown in Fig. 4(a). The degree of bending status was quantified by using the curvature unit $(k=1 / r$, where $r$ is the radius of curvature), and the electrical currents of MWCNT-CY arrays against various curvatures were measured. There was only a negligible change in the current of MWCNT-CY arrays subjected to horizontal alignment, as shown in Fig. 4(c). In contrast, substantial current variation was observed in Fig. 4(b). With increasing k (i.e., decreasing curvature radius), the value of current through perpendicularly aligned MWCNT-CY arrays decreased noticeably. The change in normalized resistance ( $R_{\text {curved }} / R_{\text {flat, }}$, where $R_{\text {flat }}$ and $R_{\text {curved }}$ indicate resistance of initially flat-positioned and bent devices, respectively) versus $\mathrm{k}$ is also plotted in the inset of Fig. 4(b). The sensitivity of bent perpendicularly aligned MWCNT-CY arrays ( $\Delta$ normalized resistance $/ \Delta \mathrm{k} \approx 1.5 \mathrm{~cm}$ ) was comparable with that of commercial sensors $(\approx 2.9 \mathrm{~cm})$ and even better than that of sensors based on three-dimensional graphite-polymer $(\approx 0.1 \mathrm{~cm}) .{ }^{[29,30]}$ Longitudinal contacts between MWCNTCYs could limit overall electrical properties in the perpendicularly aligned structure. The effective contact area of MWCNT-CYs is much smaller in bent devices than in flat-positioned devices, which increased the value of resistance in devices with high curvature, as described in supplementary Fig. S1(a). Moreover, MWCNT-CY length dominated resistance in horizontally aligned devices, and there was only a slight change in the effective length of bent MWCNT-CYs, as illustrated in supplementary Fig. S1(b).

Bending sensors (i.e., flex sensors) are widely used in human-motion detectors, electronic goniometers, and robotics. ${ }^{[30]}$ Figure 5(a) shows a Lego figure equipped with a perpendicularly aligned MWCNT-CY array (as an active bending sensor) to detect its body posture. The degree of 
Lego figure bending (from 0 degrees to 30 degrees) was successfully linked to sensor resistance variation, as shown in Fig. 5(b).

Force sensors (or pressure sensors) have many potential applications in electronic artificial skin, healthcare monitoring, biomedical devices, tactile information display, and smart shoes. ${ }^{\left[{ }^{[1-35]}\right.} \mathrm{A}$ force sensor based on the MWCNT-CY array was fabricated, as demonstrated in Fig. 6(a). The MWCNT-CY active-sensing array was placed on a glass substrate. A copper electrode attached to a flexible PET substrate was suspended on the MWCNT-CY, with a spacer as its supporting layer. When a force was applied to the flexible PET substrate, the copper electrode touched the MWCNTCY, which could modify the electrical characteristics through the active sensing element. Indeed, in the device with a perpendicularly aligned MWCNT-CY array, the electrical current was dramatically enhanced with increasing pressure, as shown in Fig. 6(b). In contrast, there was no substantial variation in the electrical current of the device based on a horizontally aligned MWCNT-CY array, shown in Fig. 6(c). Upon the application of pressure to the device in Fig. 6(b), a new parallel conducting path was created via the $\mathrm{Cu}$ electrode, and the effective contact area between MWCNT-CYs became larger (supplementary Fig. S2). These changes considerably reduced the resistance of the perpendicularly aligned MWCNT-CY device. The sensitivity (S) of force sensors can be defined as: ${ }^{[31]}$

$$
S=\frac{\delta\left[\left(R_{0}-R_{p}\right) / R_{0}\right]}{\delta P}=\frac{\delta\left(\Delta R / R_{0}\right)}{\delta P}
$$

where $\mathrm{P}, \mathrm{R}_{0}$, and $\mathrm{R}_{\mathrm{p}}$ represent the applied pressure, resistance under base pressure, and resistance when pressure is applied, respectively. For a low-pressure range $(<1 \mathrm{kPa})$, the absolute value of sensitivity reached $\approx 0.3 \mathrm{kPa}^{-1}$, but it decreased to $\approx 0.067 \mathrm{kPa}^{-1}$ with the application of greater 
force (supplementary Fig. S3). The observed sensitivity was comparable with that of CNT-based tactile sensors $\left(\approx 0.5 \mathrm{kPa}^{-1}\right)$ or graphene-based pressure sensors $\left(\approx 0.96 \mathrm{kPa}^{-1}\right) .^{[31,36]}$

Cloud computing-based IoT systems through Wi-Fi (a wireless network protocol) can resolve technical constraints related to storage, analysis, and unlimited communication in time and space of big data, which are obtained from sensor networks under various environments. ${ }^{[6-11]}$ Figure 7 shows a simple system that combines a force sensor, Arduino microcontroller, and real-time data storage in the Google Cloud platform. A force resistive sensor consisting of a perpendicularly aligned MWCNT-CY array is connected to a fixed resistor of $12 \mathrm{k} \Omega$ with voltage source (3.3 V) in series, thereby forming a voltage divider. The resistance value of the sensor device is reduced when pressure is applied, which induces variation in output voltage $\left[\mathrm{V}_{\text {out }}=12 \mathrm{k} \Omega /\right.$ (sensor resistance $+12 \mathrm{k} \Omega$ )]. An Arduino board with Wi-Fi function (Wemos D1) measures the $\mathrm{V}_{\text {out }}$ variation through the A0 port (analog in) and simultaneously sends the measured data to Google Cloud. The plot in Fig. 7 shows the sensing data (automatically stored in Google Sheets) achieved with repeated pressure (100 g force) and release operation. Actual electrical interfacing, detailed programming codes for the system, and raw data in Google Cloud are described in supplementary Fig. S4.

In addition, a proof-of-concept force sensor matrix with $5 \times 4$ pixels, based on a perpendicularly aligned MWCNT-CY array, was fabricated and demonstrated by using an Arduino uno microcontroller and 74HC4051 multiplexer, as shown in Fig. 8(a). The weight distribution on each pixel can be scanned by the sensor matrix, and the force-detecting mechanism of the pixel is identical to that in Fig. 7. The device functioned well with force $=100 \mathrm{~g}$ (supplementary Fig. S5). 
Fig. 8(b) shows the photo and contour map $\left(\mathrm{V} / \mathrm{V}_{0}\right)$ of the sensor matrix with a weight of a water bottle, where $\mathrm{V}_{0}$ and $\mathrm{V}$ denote a value of the $\mathrm{V}_{\text {out }}$ at base pressure and when a force is applied, respectively. Spatial-pressure distribution induced from the water bottle was clearly identified.

\section{Conclusions}

MWCNT-CY arrays were achieved by using a simple dipping-and-drying method without a microfabrication process or extensive chemical treatments. Perpendicularly aligned MWCNT-CY arrays contained a considerable capacitive component and resistance, but MWCNT-CY arrays with horizontal orientation possessed a resistive characteristic only. In addition, piezoresistive force and bending sensors were fabricated based on the perpendicularly aligned MWCNT-CY array, which demonstrated good performance of sensitivity $\approx 0.3 \mathrm{kPa}^{-1}$ and $\Delta$ normalized resistance/ $\Delta$ curvature $\approx 1.5 \mathrm{~cm}$. A simple system, consisting of a perpendicularly aligned MWCNT-CY array-based force

sensor, microcontroller with Wi-Fi module (Arduino Wemos D1), and real-time data storage in Google Cloud platform, was also successfully demonstrated. Moreover, a proof-of-concept force sensor matrix $(5 \times 4$ pixels $)$ was constructed with a contour plot that clearly showed spatialpressure distribution.

\section{Experimental details}

Pristine cotton yarns were dipped into $0.3 \mathrm{wt} \%$ MWCNT water dispersion (MWCNTs; diameter $=5$ to $15 \mathrm{~nm}$, length $\approx 50 \mu \mathrm{m}$; US Research Nanomaterials, Inc.) for 1 week, then dried for $\approx 24 \mathrm{~h}$ at room temperature. A uniformly MWCNT-coated cotton yarn was obtained without any sonication or chemical treatment. Raman spectra at a laser wavelength of $514 \mathrm{~nm}$ were obtained by using a LabRAM micro-Raman from HORIBA. Impedance characteristics of MWCNT-CY 
arrays were recorded using a HP4192A impedance analyzer, and a Keithley 2400 measurement unit was used for bending analysis with an interface programmed by LABVIEW software. Arduino boards (Uno and Wemos D1) were used as microcontrollers for the demonstration of a force sensor system equipped with Wi-Fi communication, as well as a sensor matrix. Actual electrical connections and detail programming codes for Arduino and Google Sheets are described in supplementary Figs. S4 and S5.

\section{Acknowledgments}

This work was supported by the National Research Foundation of Korea (NRF) (2017M3A7B4049167) and the Korea Institute of Science and Technology (KIST), Open Research Program.

\section{Author contributions}

D.-Y.J. supervised the research and wrote the manuscript. D.-Y.J. and T.Y.L. performed the experiments, data analysis and interpretation. D.-Y.J., S.J.P. and G.T.K. designed and implemented the IoT sensing systems. All authors reviewed the manuscript.

\section{Competing interests}

The authors declare no competing interests. 


\section{FIGURE CAPTIONS}

Figure 1. (a) MWCNT-coated yarn (CY) prepared by a simple dipping-and-drying method, and scanning electron microscope (SEM) image of the resulting MWCNT-CY. (b) Raman spectrum of the MWCNT-CY showing two clear peaks (laser wavelength $=514 \mathrm{~nm}$ ).

Figure 2. Aligned MWCNT-CY array with two different orientations (perpendicularly or horizontally aligned to the electrodes) on PET substrate.

Figure 3. Total impedance $(Z)$ and phase angle $(\theta)$ measured from (a) a perpendicularly aligned MWCNT-CY array, and (b) a horizontally aligned MWCNT-CY array. Schematic architectures describe the structures of devices with the expected electrical parameters.

Figure 4. (a) Illustration describing the relation between curvature (k) and radius of curvature (r), with the MWCNT-CY array device easily bent as shown in photos. (b) Measured current versus applied voltage with varying $\mathrm{k}$ in the perpendicularly aligned MWCNT-CY array device, with inset showing normalized resistance versus $\mathrm{k}$ to extract a sensitivity value. (c) Current versus voltage with varying $\mathrm{k}$ in the horizontally aligned MWCNT-CY array device.

Figure 5. (a) Lego figure equipped with the bending sensor based on a perpendicularly aligned MWCNT-CY array as an active sensing material. (b) Measured resistance of bending sensor with varying degree of Lego figure bending.

Figure 6. (a) Schematic architecture of device structure of a force sensor based on a MWCNT-CY array. Measured current versus applied voltage with varying pressure in the force sensor with (b) a perpendicularly aligned MWCNT-CY array, or (c) a horizontally aligned MWCNT-CY array. Plots in the insets show resistance variation versus pressure. 
Figure 7. Simple system consisting of a force sensor, Arduino microcontroller, and real-time data storage in Google Cloud platform. The force sensor was based on a perpendicularly aligned MWCNT-CY array, and the plot shows the sensing data (automatically stored in Google Sheets) generated under repeated pressure and release operation.

Figure 8. (a) System structure and detail electrical connections for a force sensor matrix with $5 \times$ 4 pixels. (b) Photo and contour map $\left(\mathrm{V} / \mathrm{V}_{0}\right)$ of a force sensor matrix with the weight of a water bottle. 


\section{REFERENCES}

(1) Yeo, J. C. \& Lim, C. T. Emerging flexible and wearable physical sensing platforms for healthcare and biomedical applications. Microsystems \& Nanoengineering 2, 1-19 (2016).

(2) Amjadi, M., Kyung, K. U., Park, I. \& Sitti, M. Stretchable, skin-mountable, and wearable strain sensors and their potential applications: a review. Advanced Functional Materials 26, 1678-1698 (2016).

(3) Cardenas, J. A., Andrews, J. B., Noyce, S. G. \& Franklin, A. D. Carbon nanotube electronics for IoT sensors. Nano Futures 4, 012001 (2020).

(4) Yu, Y., Nyein, H. Y. Y., Gao, W. \& Javey, A. Flexible electrochemical bioelectronics: the rise of in situ bioanalysis. Advanced Materials 32, 1902083 (2020).

(5) Souri, H. et al. Wearable and Stretchable Strain Sensors: Materials, Sensing Mechanisms, and Applications. Advanced Intelligent Systems, 2000039 (2020).

(6) Din, I. U. et al. The Internet of Things: A review of enabled technologies and future challenges. Ieee Access 7, 7606-7640 (2018).

(7) Gil, D., Ferrández, A., Mora-Mora, H. \& Peral, J. Internet of things: A review of surveys based on context aware intelligent services. Sensors 16, 1069 (2016).

(8) Ng, I. C. \& Wakenshaw, S. Y. The Internet-of-Things: Review and research directions. International Journal of Research in Marketing 34, 3-21 (2017).

(9) Kim, S.-M., Choi, Y. \& Suh, J. Applications of the Open-Source Hardware Arduino Platform in the Mining Industry: A Review. Applied Sciences 10, 5018 (2020).

(10) Heradio, R. et al. Open-source hardware in education: A systematic mapping study. Ieee Access 6, 72094-72103 (2018). 
(11) Park, S. J., Jeon, D.-Y., Moon, Y.-S., Park, I.-H. \& Kim, G.-T. Web-drive based source measure unit for automated evaluations of ionic liquid-gated MoS2 transistors. Review of Scientific Instruments 90, 124708 (2019).

(12) De Volder, M. F., Tawfick, S. H., Baughman, R. H. \& Hart, A. J. Carbon nanotubes: present and future commercial applications. science 339, 535-539 (2013).

(13) Javey, A. \& Kong, J. Carbon nanotube electronics. (Springer Science \& Business Media, 2009).

(14) Suzuki, K. et al. Rapid-response, widely stretchable sensor of aligned MWCNT/elastomer composites for human motion detection. Acs Sensors 1, 817-825 (2016).

(15) Yamada, T. et al. A stretchable carbon nanotube strain sensor for human-motion detection. Nature nanotechnology 6, 296 (2011).

(16) Zhao, Y. et al. Single-wall carbon nanotube-coated cotton yarn for electrocardiography transmission. Micromachines 9, 132 (2018).

(17) Yang, M. et al. Conductive cotton fabrics for motion sensing and heating applications. Polymers 10, 568 (2018).

(18) $\mathrm{Hu}$, L. et al. Stretchable, porous, and conductive energy textiles. Nano letters 10, 708-714 (2010).

(19) Liu, M. et al. Large-area all-textile pressure sensors for monitoring human motion and physiological signals. Advanced Materials 29, 1703700 (2017).

(20) Dhanabalan, S. C., Dhanabalan, B., Chen, X., Ponraj, J. S. \& Zhang, H. Hybrid carbon nanostructured fibers: Stepping stone for intelligent textile-based electronics. Nanoscale 11, 3046-3101 (2019). 
(21) Murphy, H., Papakonstantinou, P. \& Okpalugo, T. T. Raman study of multiwalled carbon nanotubes functionalized with oxygen groups. Journal of Vacuum Science \& Technology B: Microelectronics and Nanometer Structures Processing, Measurement, and Phenomena 24, 715-720 (2006).

(22) Chinh, V. D. et al. Synthesis of gold nanoparticles decorated with multiwalled carbon nanotubes (Au-MWCNTs) via cysteaminium chloride functionalization. Scientific reports 9, $1-9$ (2019).

(23) Okada, K. \& Sekino, T. Impedance Measurement Handbook. Agilent Technologies 128, 5950-3000 (2003).

(24) Van Gerwen, P. et al. Nanoscaled interdigitated electrode arrays for biochemical sensors. Sensors and Actuators B: Chemical 49, 73-80 (1998).

(25) Jeon, D.-Y. et al. Impedance characterization of nanogap interdigitated electrode arrays fabricated by tilted angle evaporation for electrochemical biosensor applications. Journal of Vacuum Science \& Technology B, Nanotechnology and Microelectronics: Materials, Processing, Measurement, and Phenomena 32, 021803 (2014).

(26) Sanli, A., Müller, C., Kanoun, O., Elibol, C. \& Wagner, M. F.-X. Piezoresistive characterization of multi-walled carbon nanotube-epoxy based flexible strain sensitive films by impedance spectroscopy. Composites Science and Technology 122, 18-26 (2016).

(27) Jeon, D.-Y., Kim, H., Lee, M. W., Park, S. J. \& Kim, G.-T. Piezo-impedance response of carbon nanotube/polydimethylsiloxane nanocomposites. APL Materials 7, 041118 (2019).

(28) Nam, D., Jung, M., Ahn, S.-e. \& Jeon, D.-Y. Orientation-dependent impedance response of highly aligned carbon nanotube sheets. Sensors and Actuators A: Physical 313, 112187 (2020). 
(29) Li, W., Guo, J. \& Fan, D. 3D graphite-polymer flexible strain sensors with Ultrasensitivity and durability for real-time human vital sign monitoring and musical instrument education. Advanced Materials Technologies 2, 1700070 (2017).

(30) Saggio, G. \& Orengo, G. Flex sensor characterization against shape and curvature changes. Sensors and Actuators A: Physical 273, 221-231 (2018).

(31) Tian, H. et al. A graphene-based resistive pressure sensor with record-high sensitivity in a wide pressure range. Scientific reports 5, 8603 (2015).

(32) Someya, T. et al. A large-area, flexible pressure sensor matrix with organic field-effect transistors for artificial skin applications. Proceedings of the National Academy of Sciences 101, 9966-9970 (2004).

(33) Pan, L. et al. An ultra-sensitive resistive pressure sensor based on hollow-sphere microstructure induced elasticity in conducting polymer film. Nature communications 5, 1-8 (2014).

(34) Momin, M. A., Rahman, M. J. \& Mieno, T. Foot pressure sensor system made from MWCNT coated cotton fibers to monitor human activities. Surface and Coatings Technology, 125749 (2020).

(35) Eskofier, B. M. et al. An overview of smart shoes in the internet of health things: gait and mobility assessment in health promotion and disease monitoring. Applied Sciences 7, 986 (2017).

(36) Kim, S. Y. et al. Highly sensitive and multimodal all-carbon skin sensors capable of simultaneously detecting tactile and biological stimuli. Advanced Materials 27, 4178-4185 (2015). 
The English in this document has been checked by at least two professional editors, both native speakers of English. For a certificate, please see:

http://www.textcheck.com/certificate/njdf3P 


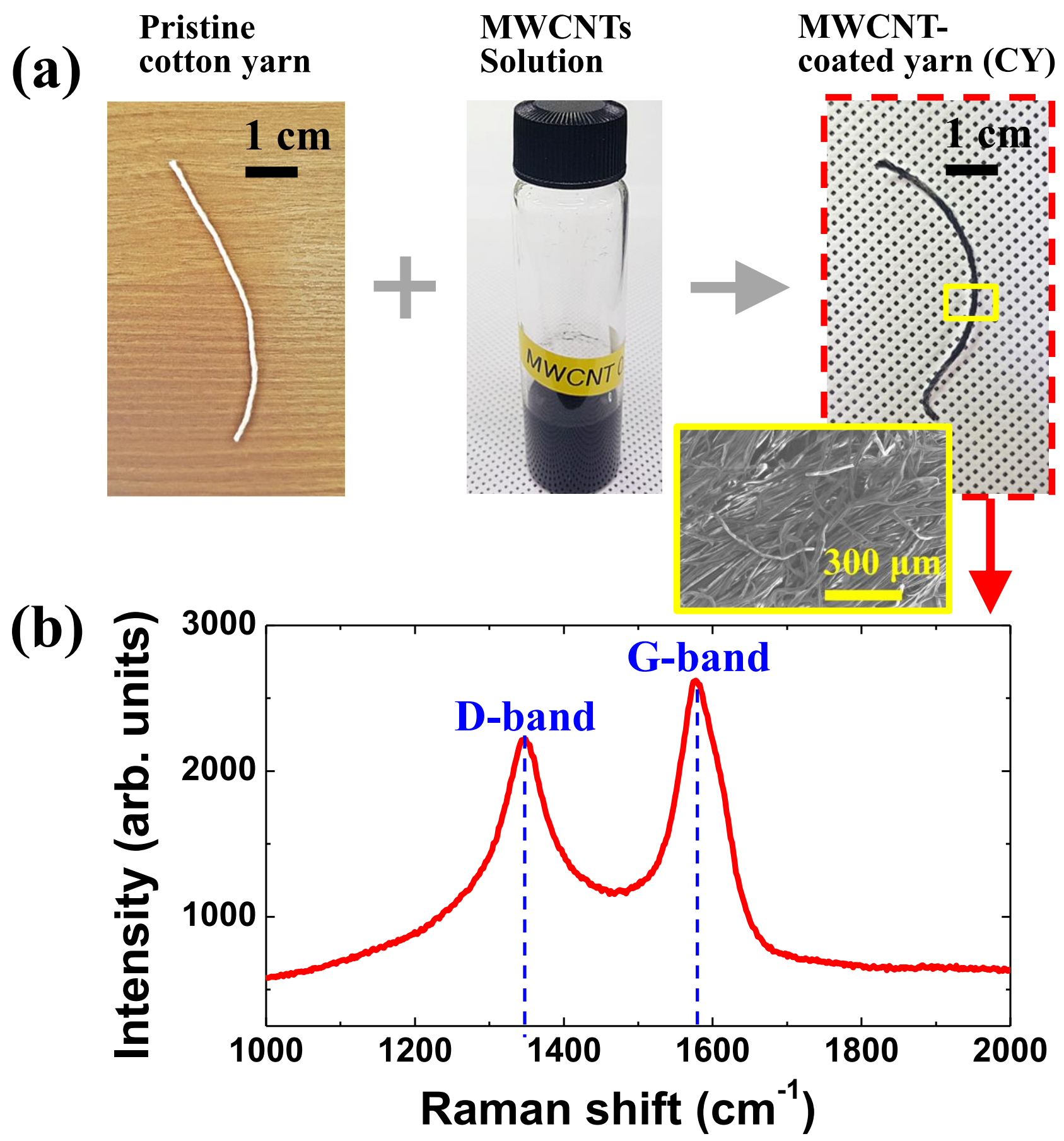

Fig. 1 D. - Y. Jeon et al. 


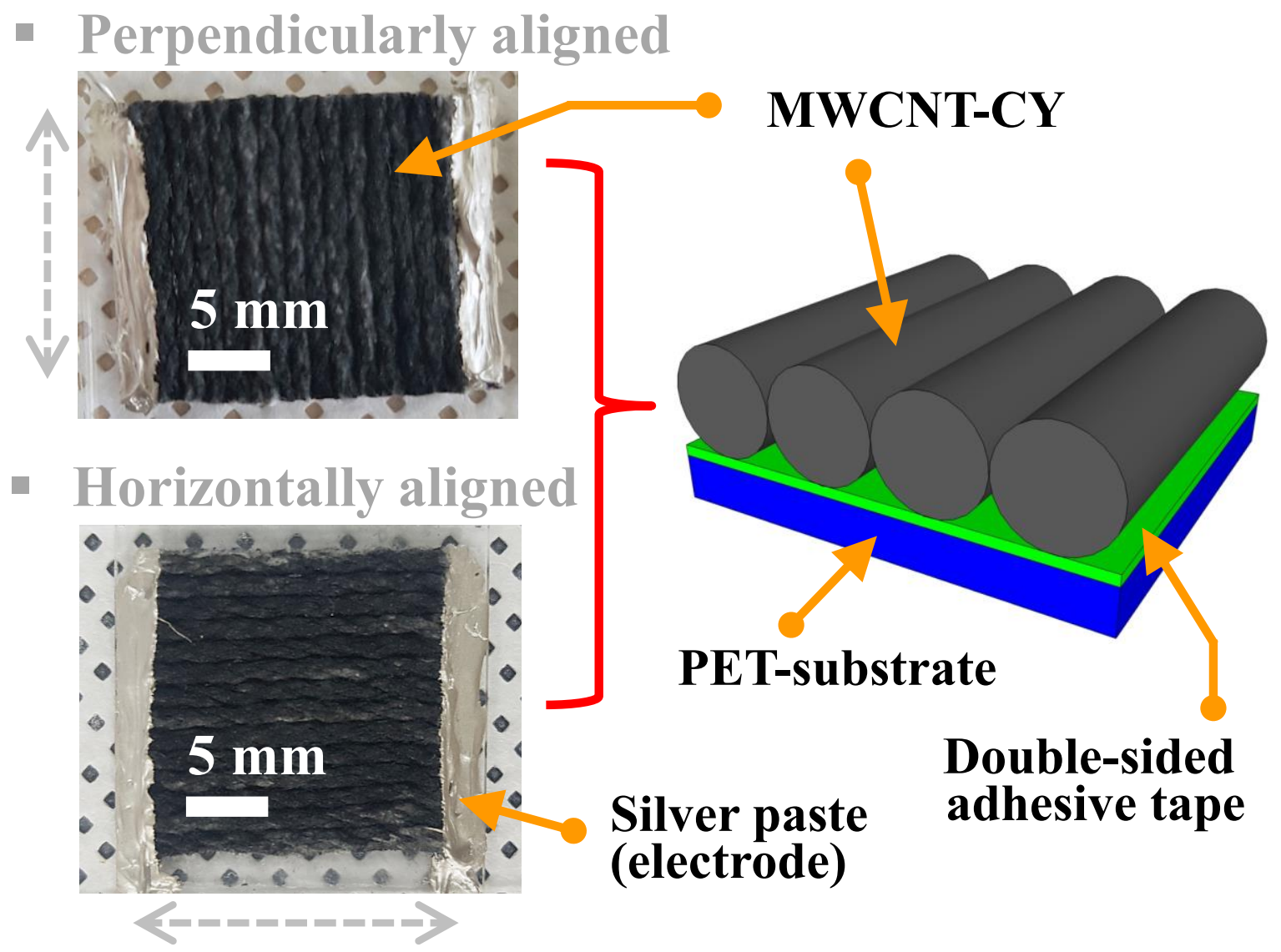



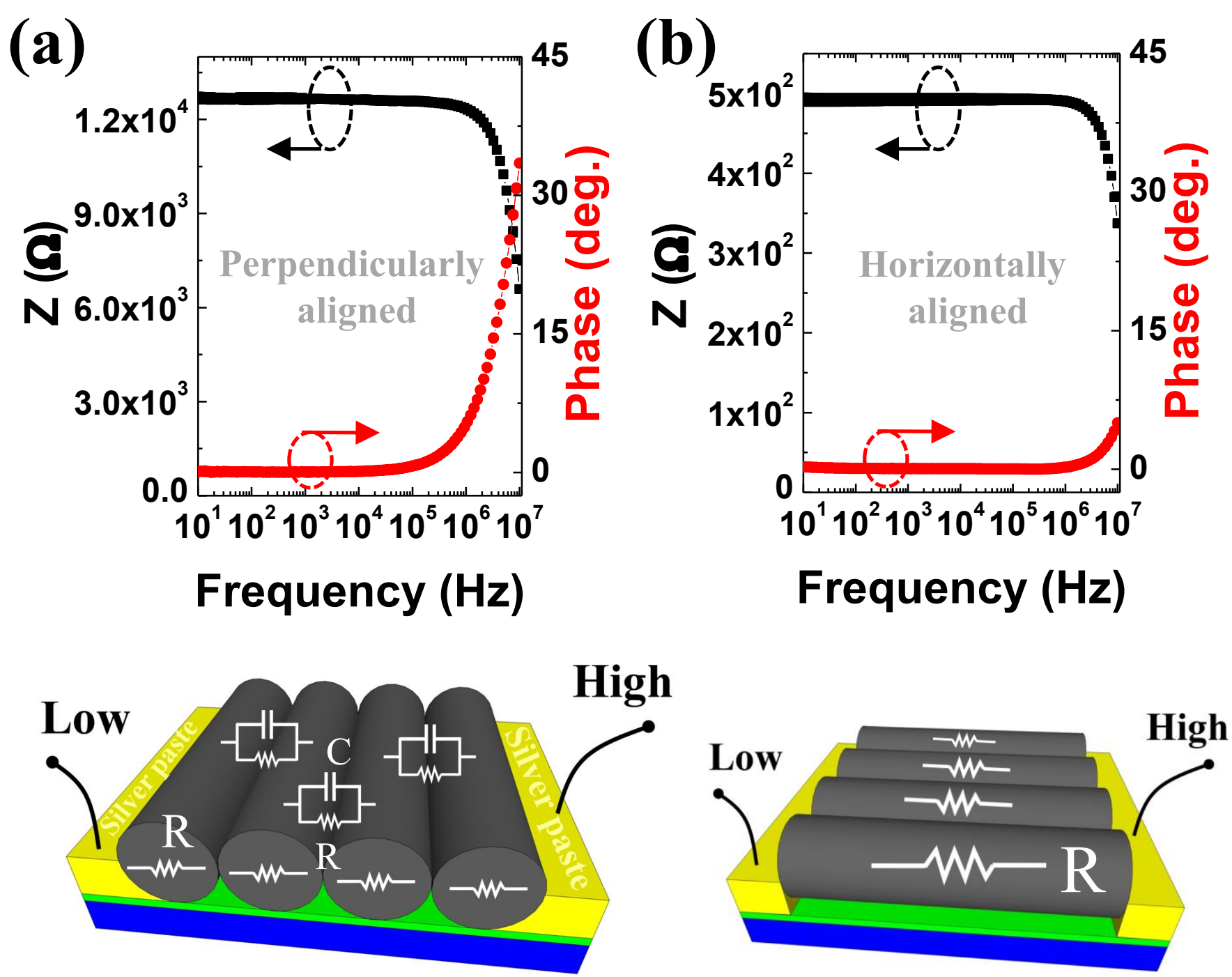

$\checkmark$ Considerable capacitance and resistance 
(a)

$\mathbf{k}=\mathbf{0}(\mathbf{r}=\infty \mathbf{c m}$, flat $)$
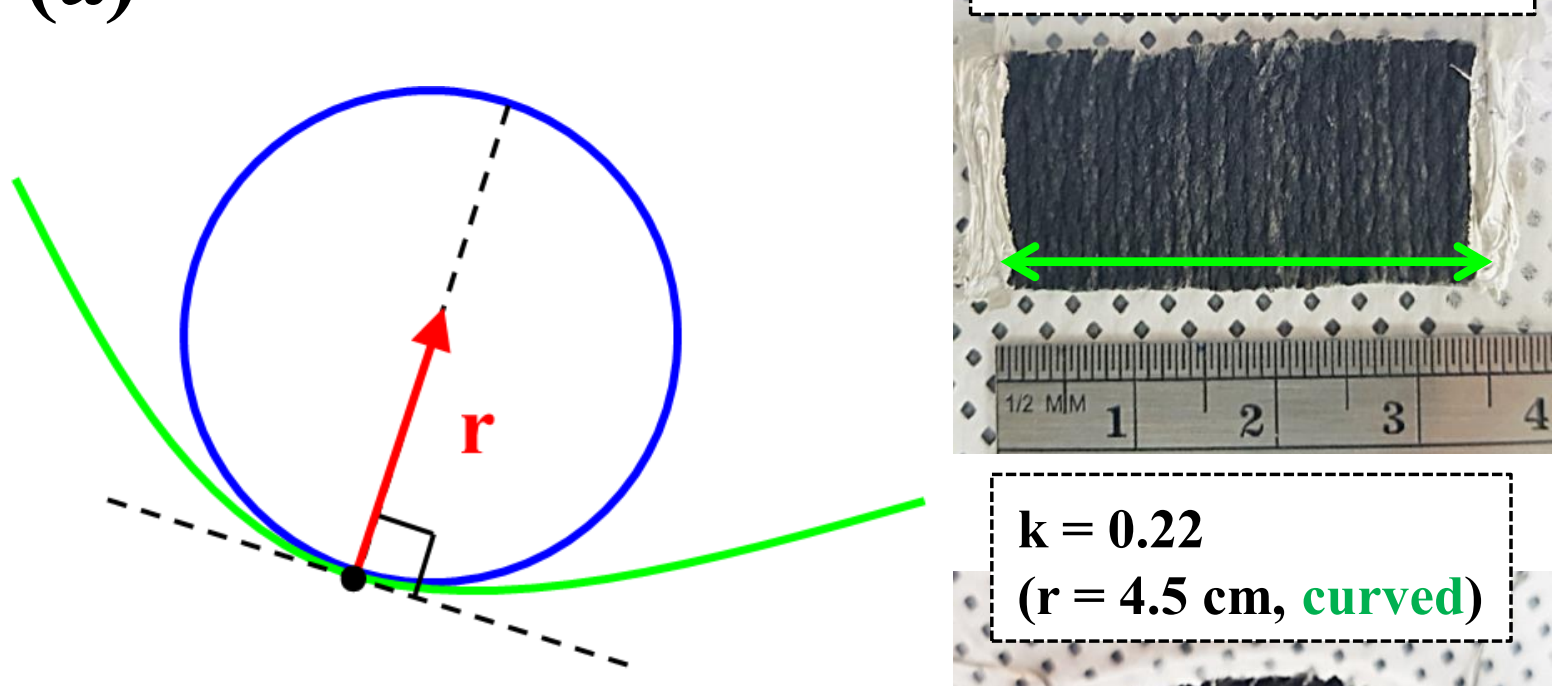

$k=0.22$

( $r=4.5 \mathrm{~cm}$, curved)

- Curvature: $\mathrm{k}=1 / \mathrm{r}$

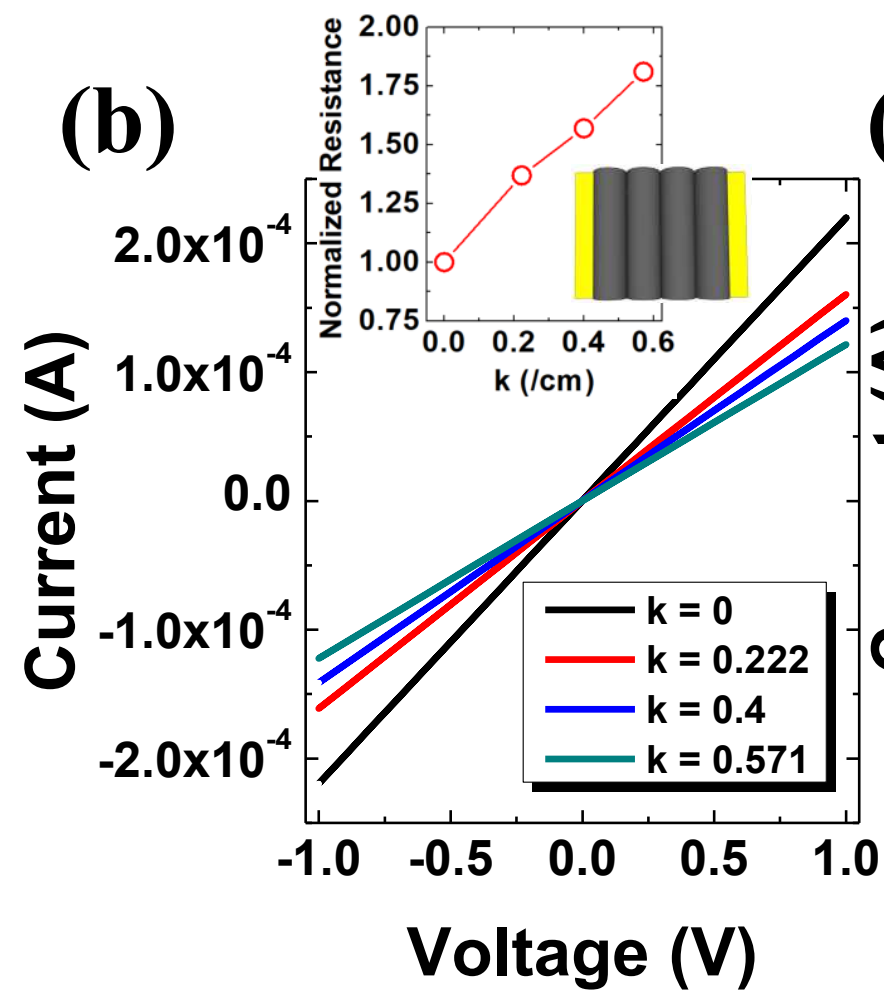

(c)

* Perpendicullarly aligned

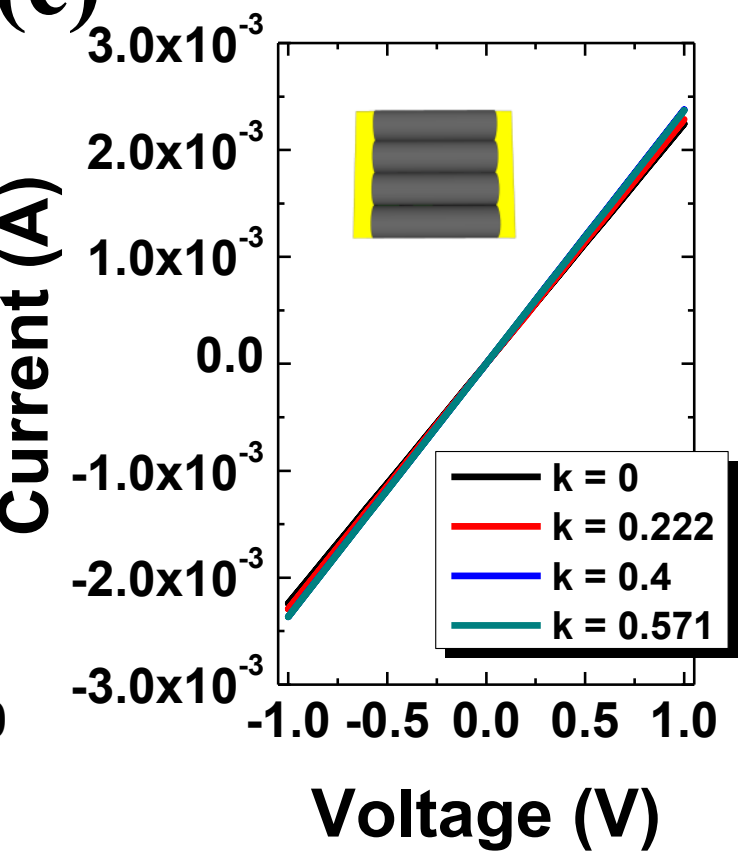

Fig. 4 D. - Y. Jeon et al. 


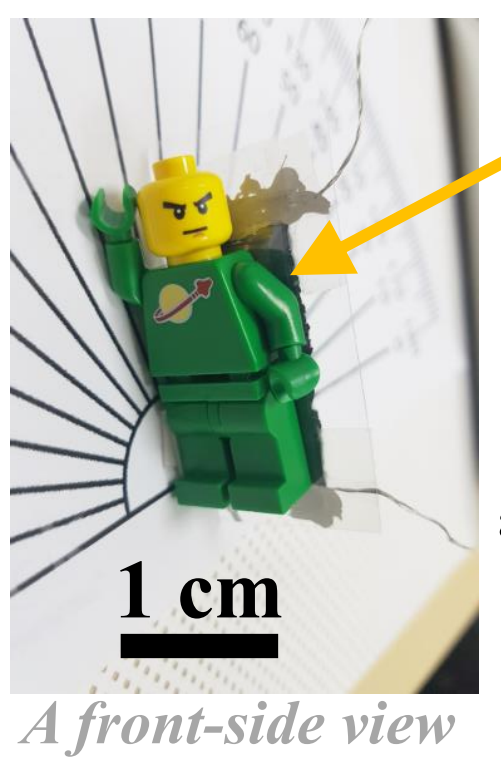

Bending sensor attached to the back of a Lego figure

Perpendicularly aligned MWCNT-CY

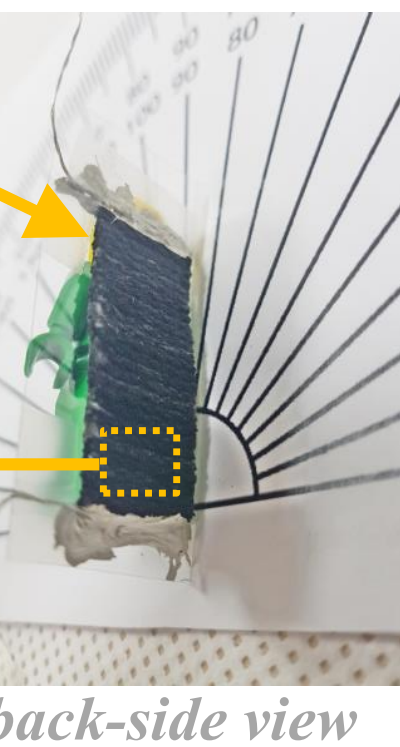

(b)
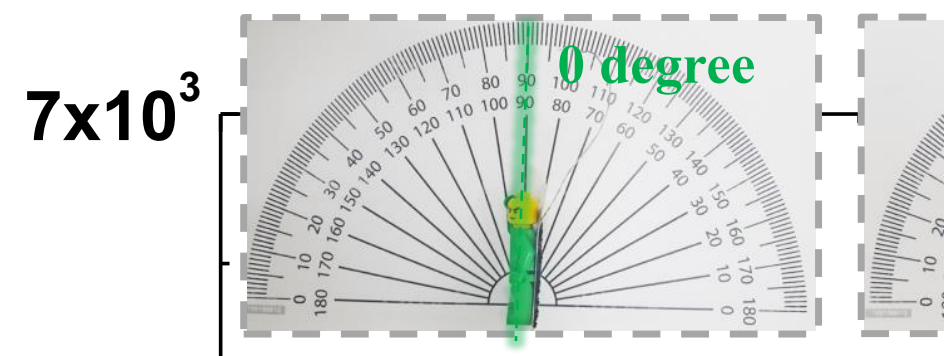

A back-side view
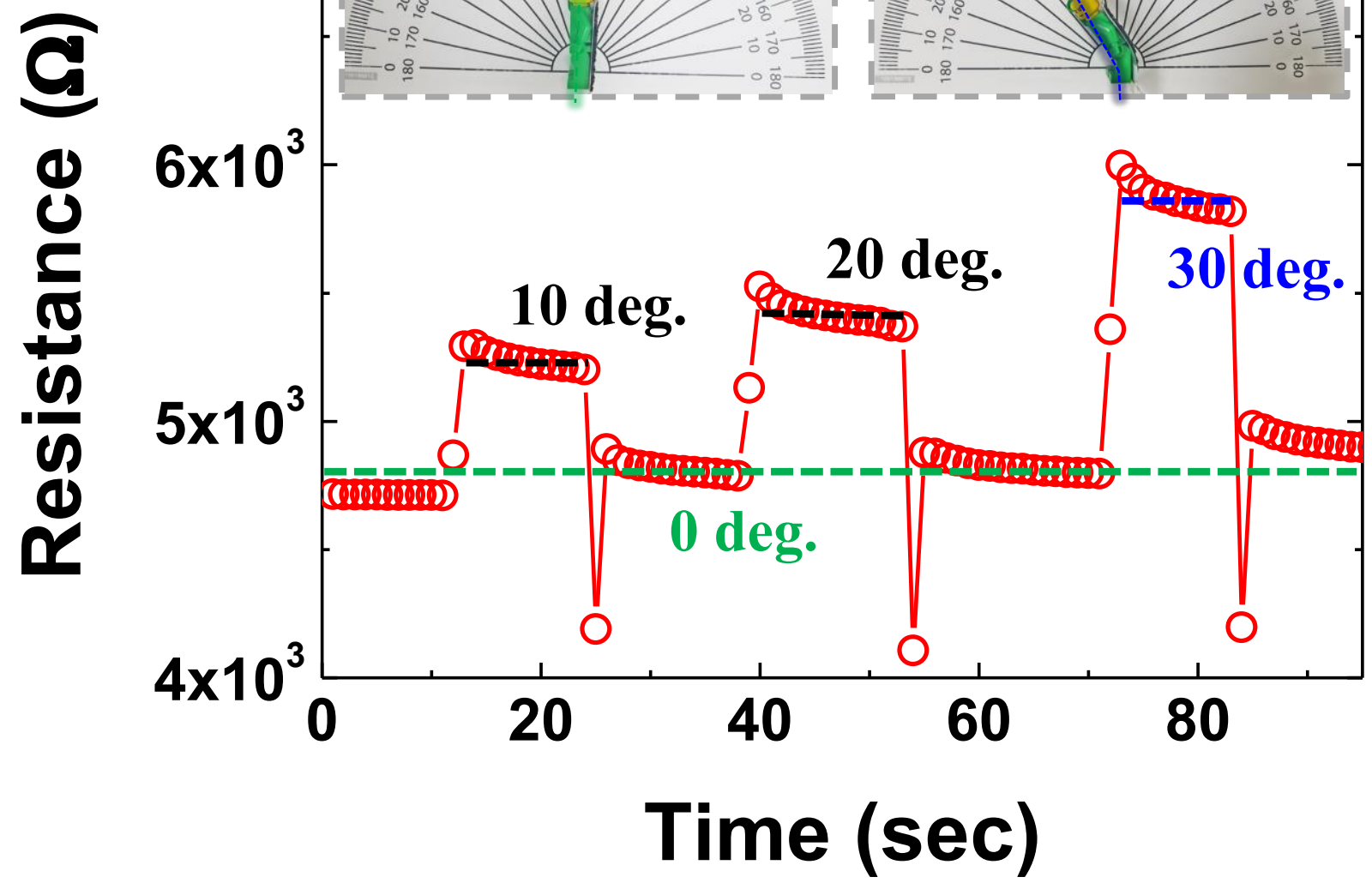

Fig. 5 D. - Y. Jeon et al. 
(a)

$<$ No pressure $>$

Electrode

(Silver paste)

Glass-substrate
(Perpendicularly aligned

or Horizontally aligned)

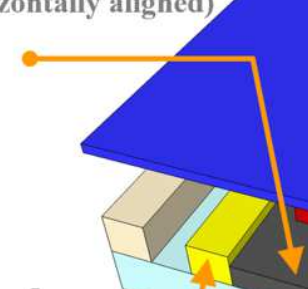

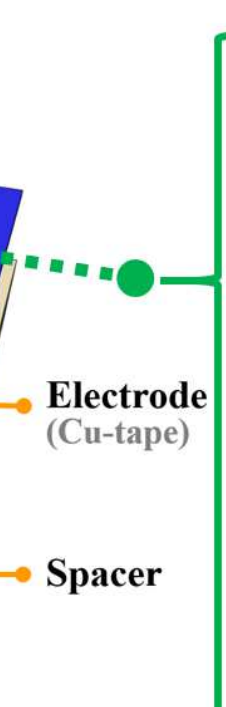

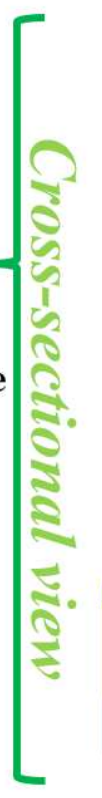

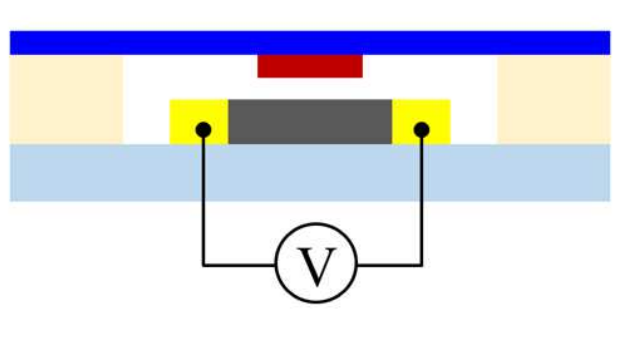

\section{$<$ Under pressure $>$}

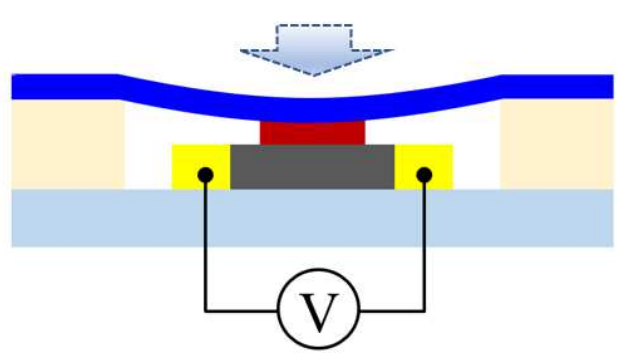

(b) Perpendicularly aligned
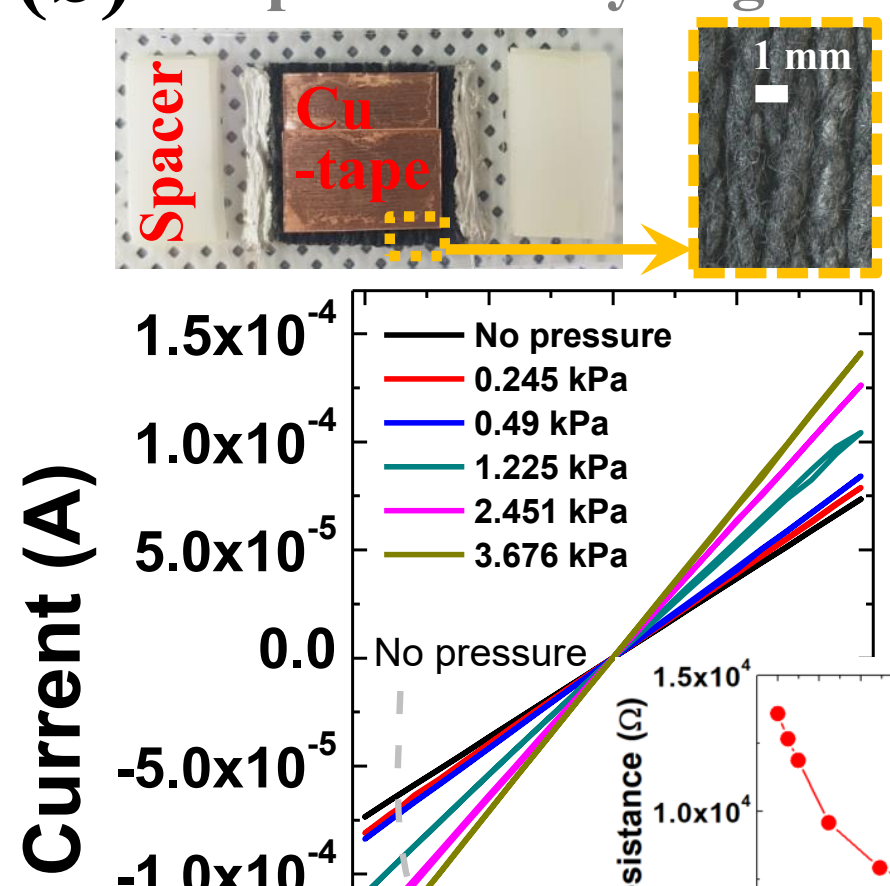

(c)

Horizontally aligned
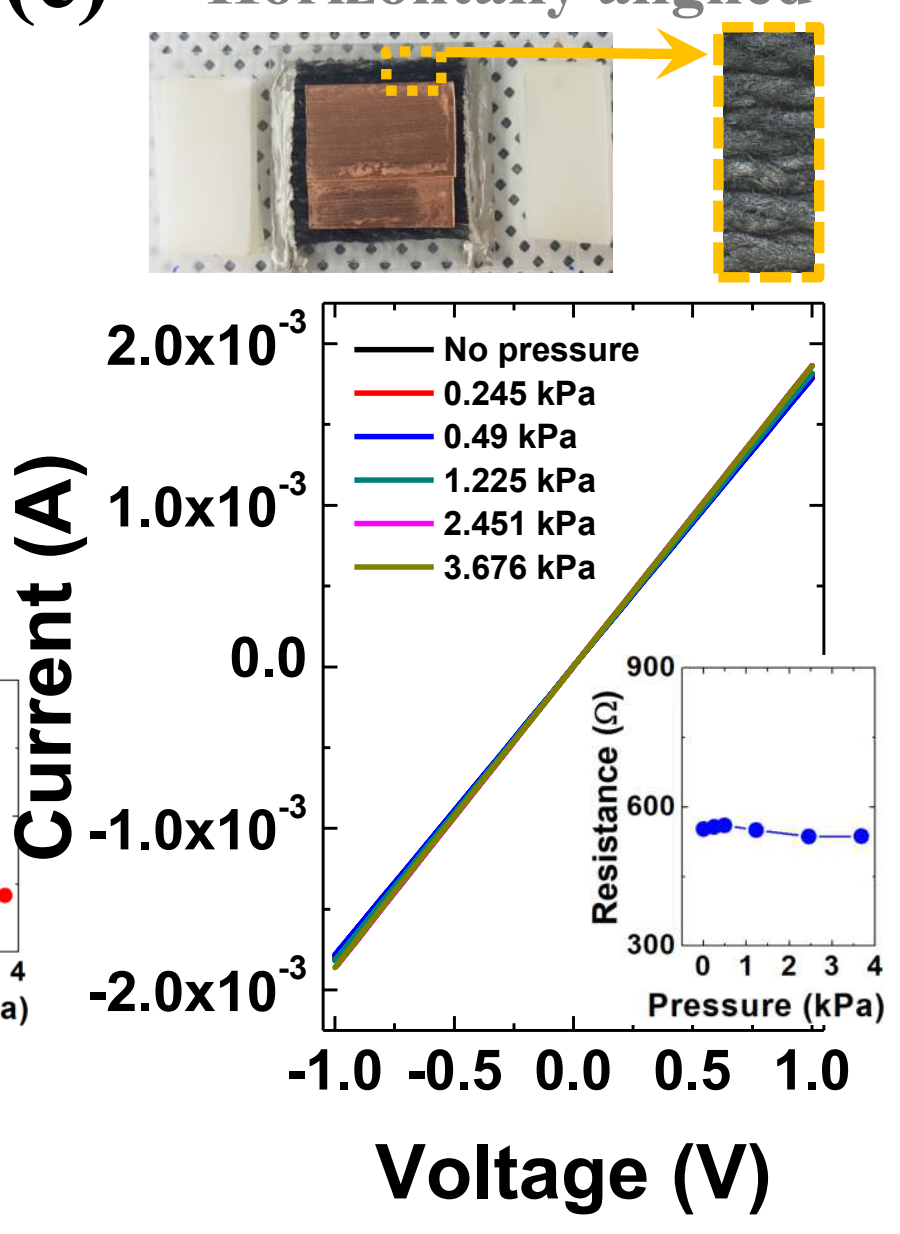

Fig. 6 D. -Y. Jeon et al. 


\section{Force sensor}

with perpendicularly aligned MWCNT-CY
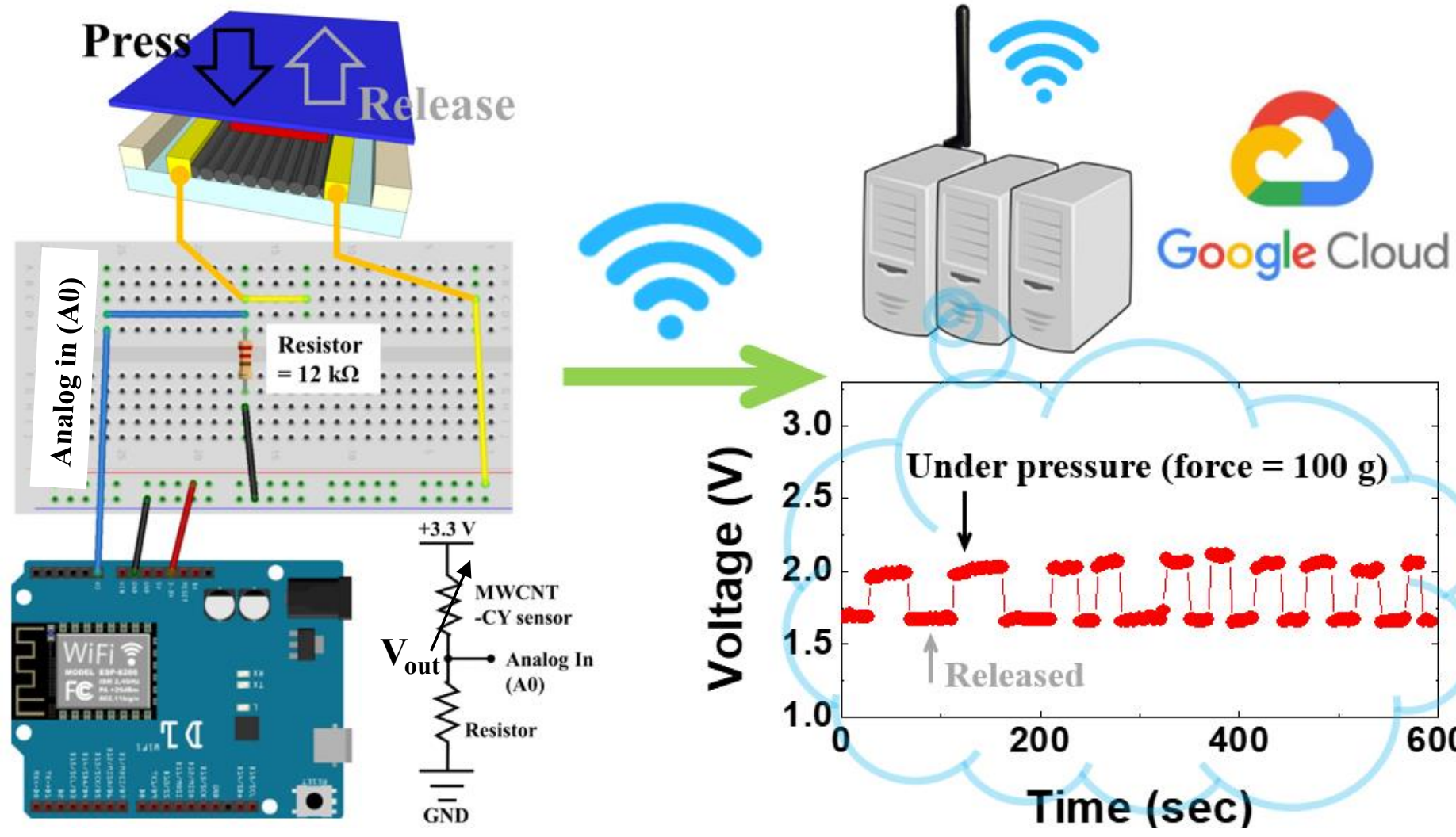

Arduino board (Wemos D1 wifi)

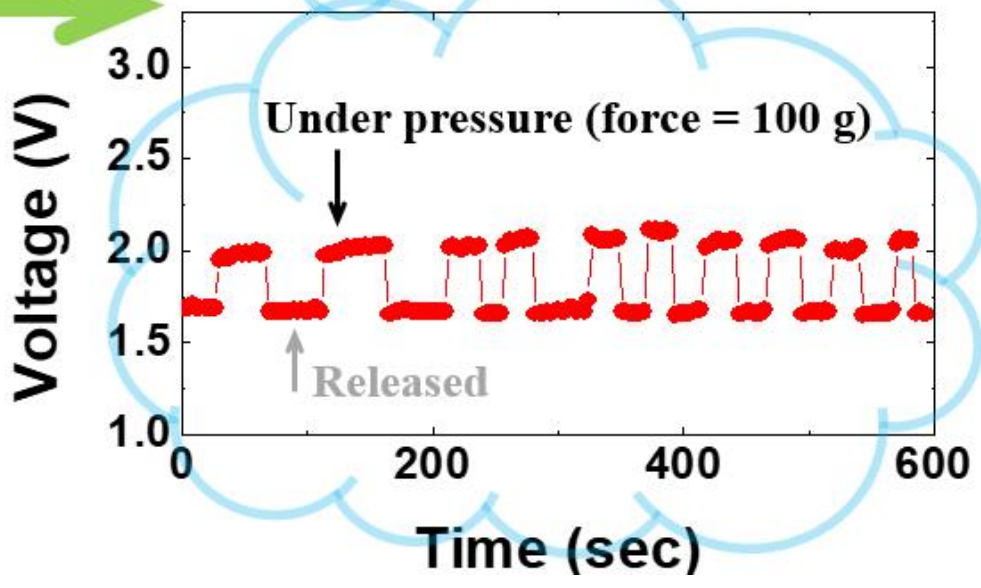


(a)

Analog In (A0)

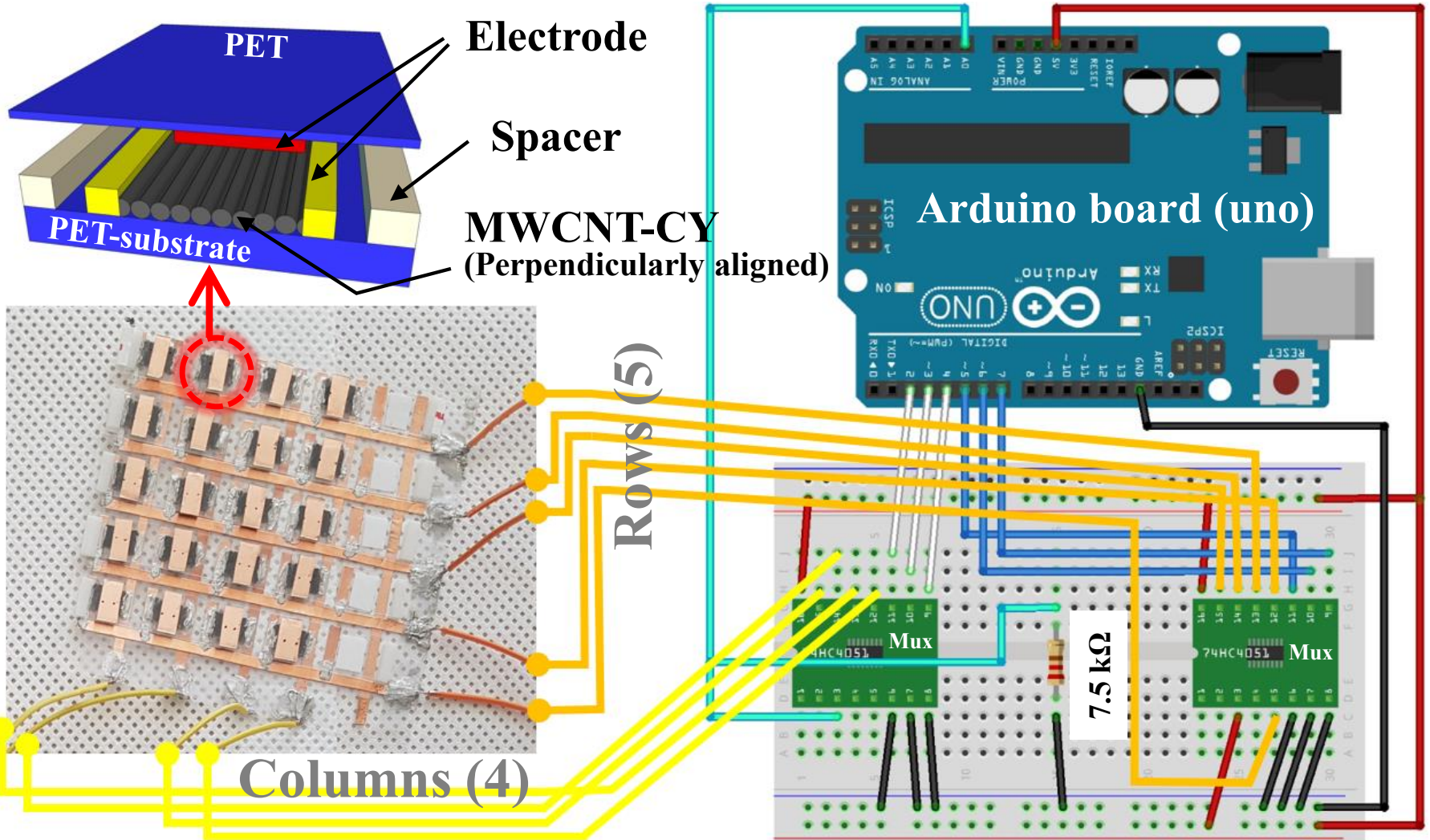

(b)
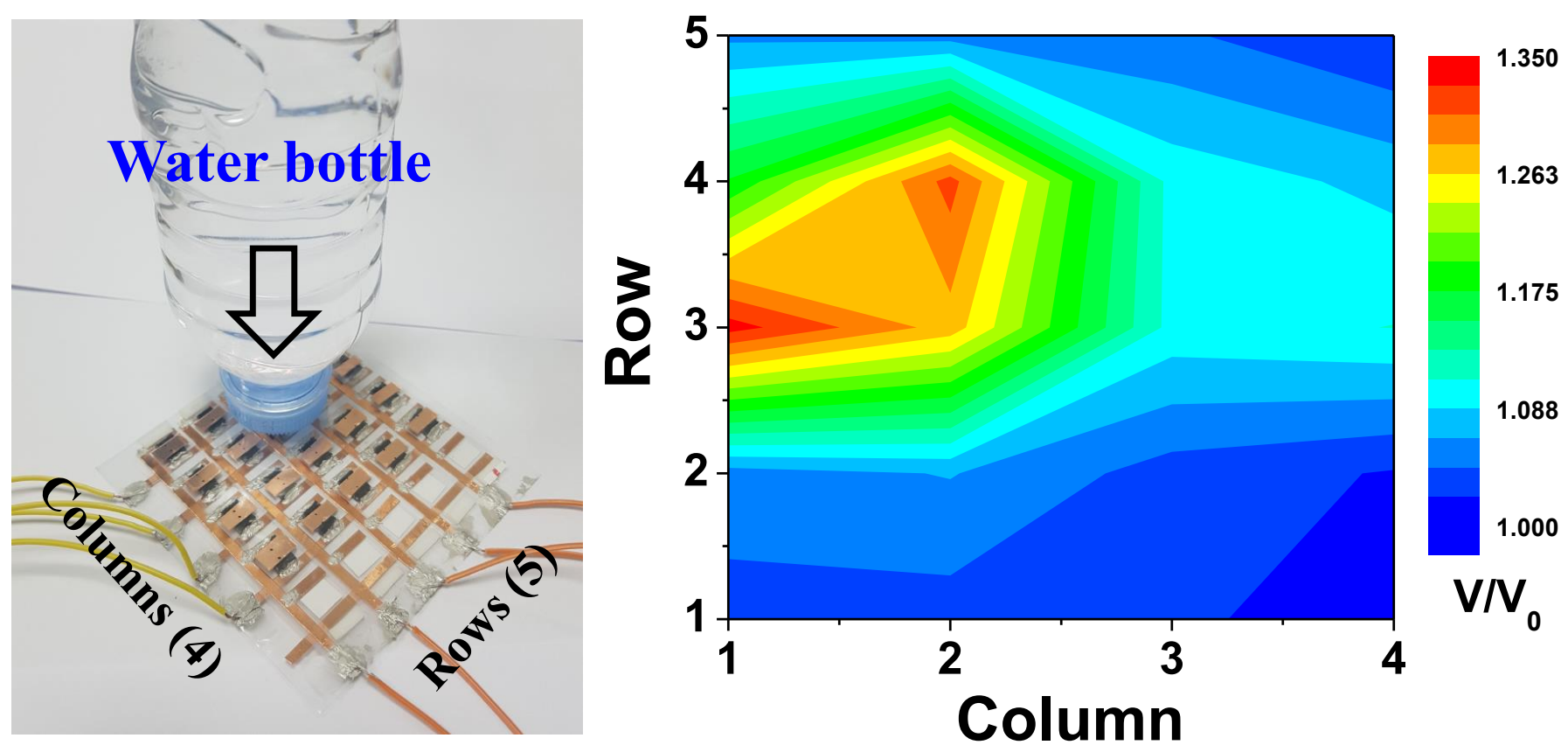

Fig. 8 D. -Y. Jeon et al. 
Figures

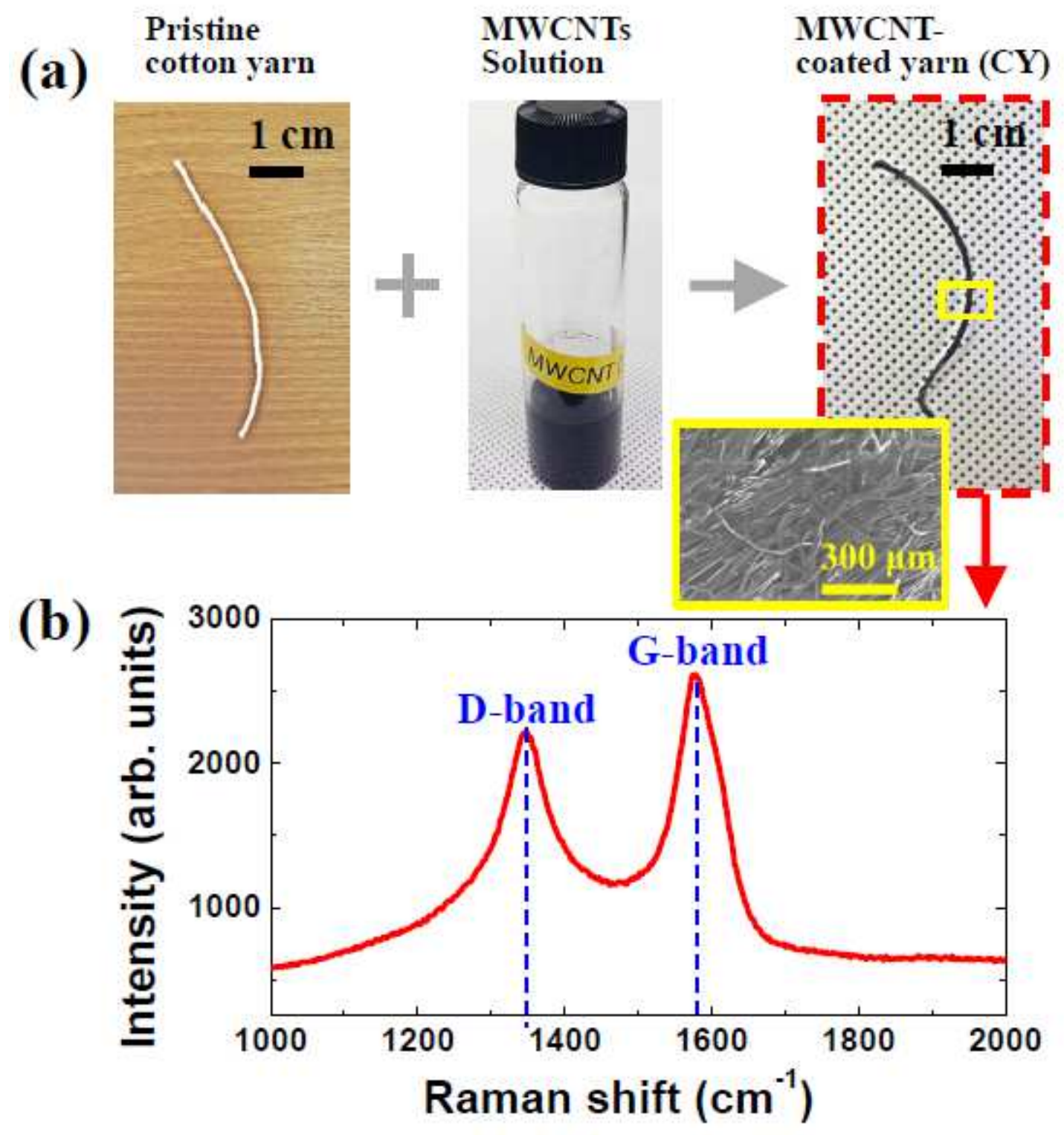

Figure 1

(a) MWCNT-coated yarn (CY) prepared by a simple dipping-and-drying method, and scanning electron microscope (SEM) image of the resulting MWCNT-CY. (b) Raman spectrum of the MWCNT-CY showing two clear peaks (laser wavelength $=514 \mathrm{~nm}$ ). 
- Perpendicularly aligned

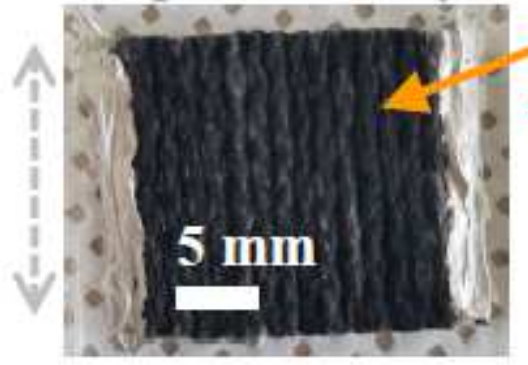

- Horizontally aligned

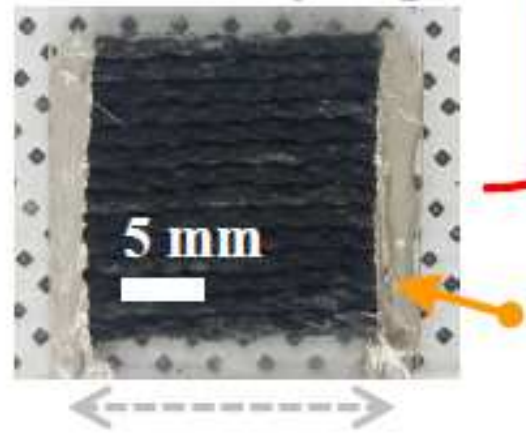

\section{MWCNT-CY}

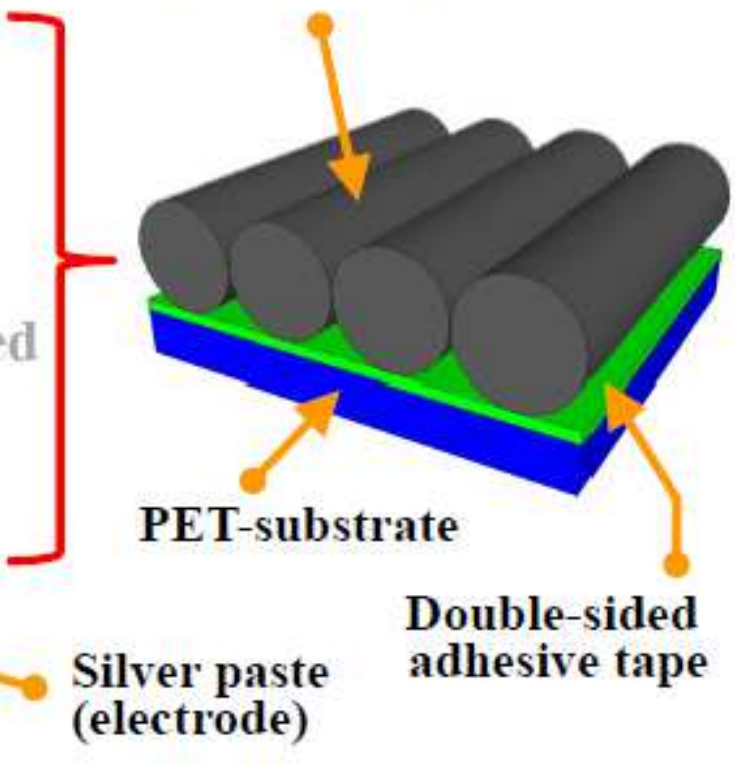

Figure 2

Aligned MWCNT-CY array with two different orientations (perpendicularly or horizontally aligned to the electrodes) on PET substrate. 

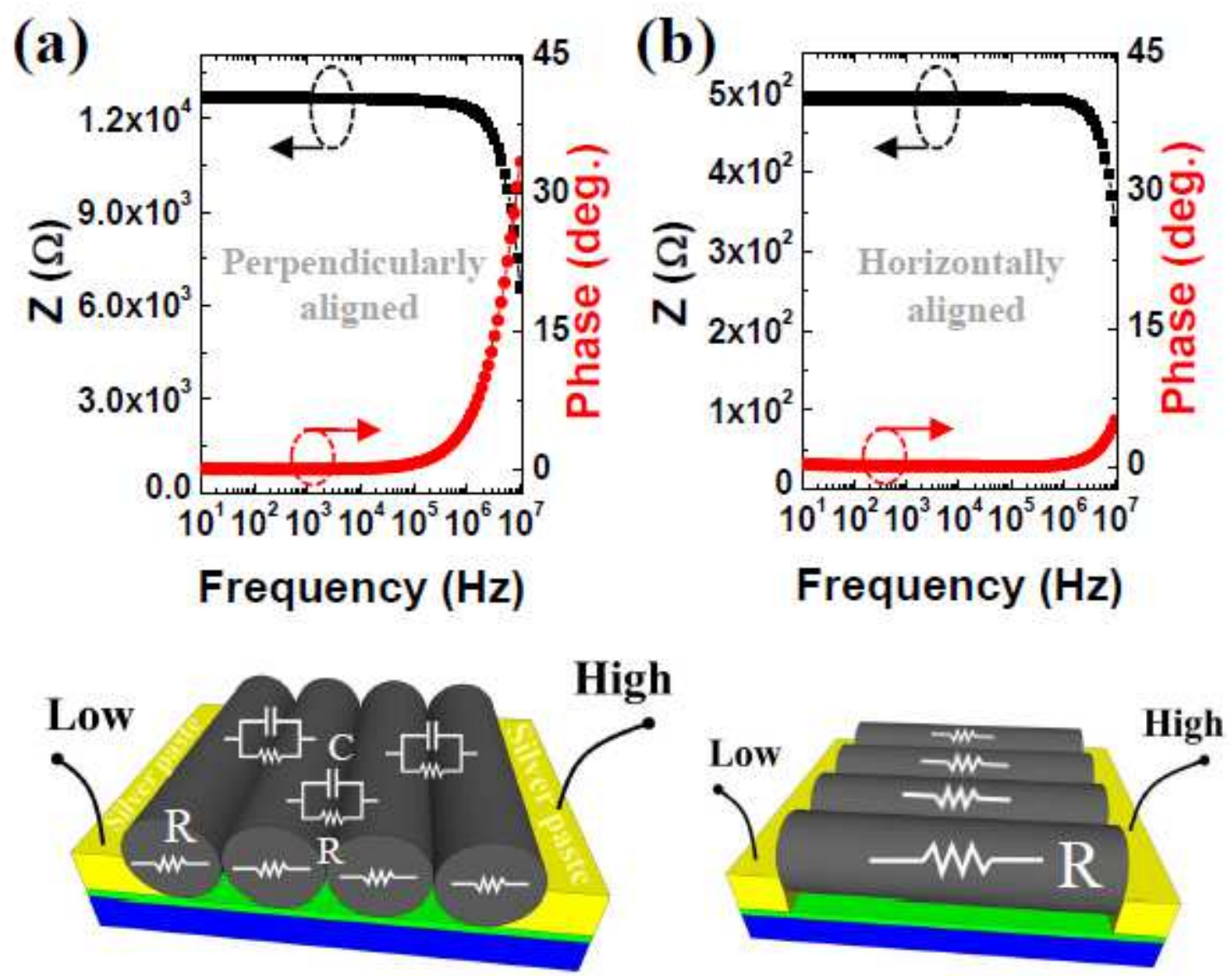

\section{$\checkmark$ Considerable capacitance and resistance}

Figure 3

Total impedance (Z) and phase angle ( $\theta$ ) measured from (a) a perpendicularly aligned MWCNT-CY array, and (b) a horizontally aligned MWCNT-CY array. Schematic architectures describe the structures of devices with the expected electrical parameters. 

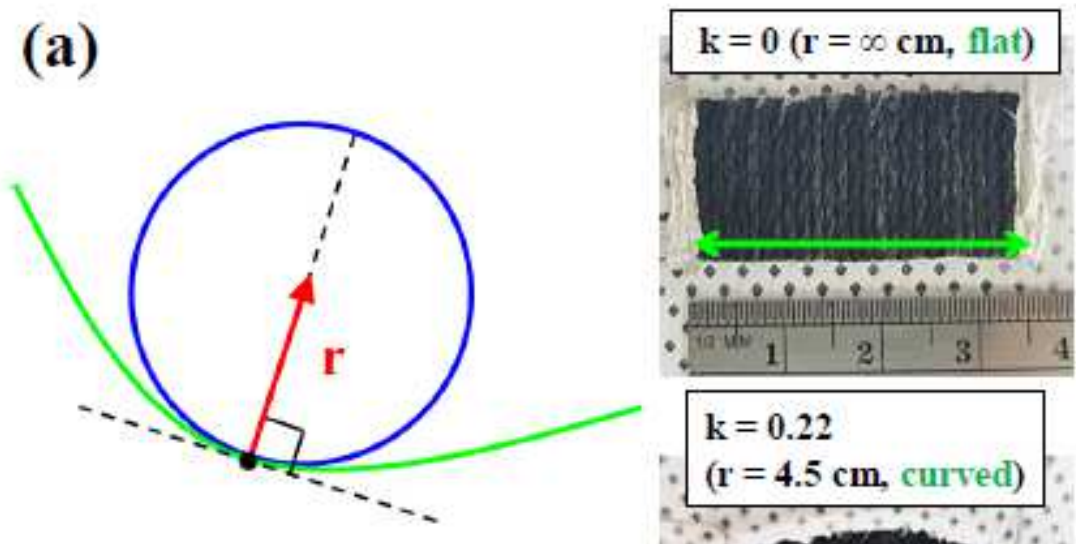

- Curvature: $\mathrm{k}=1 / \mathrm{r}$

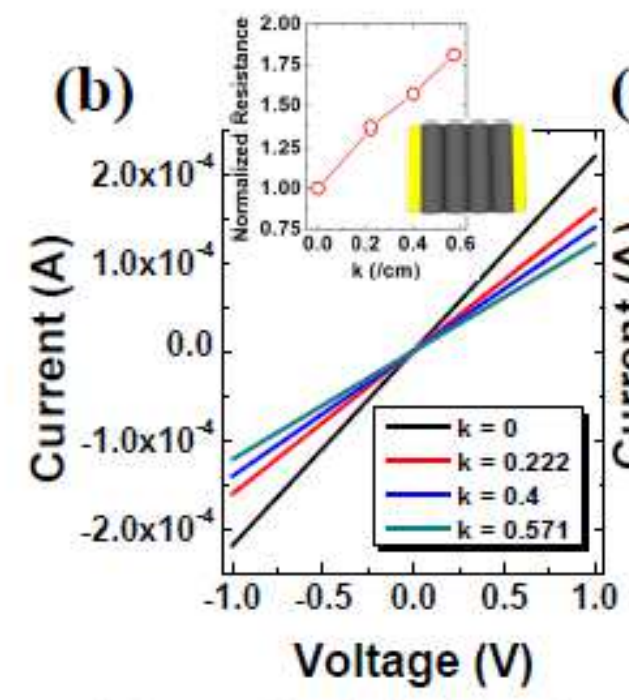

$\%$ Perpendicularly aligned

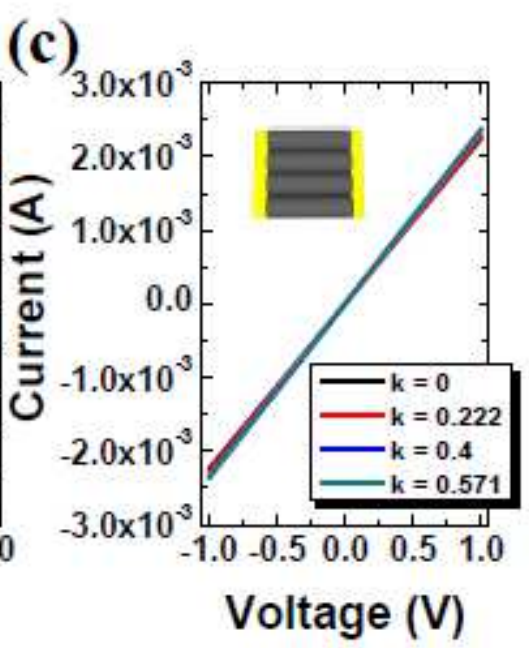

* Horizontally aligned

\section{Figure 4}

(a) Illustration describing the relation between curvature (k) and radius of curvature (r), with the MWCNTCY array device easily bent as shown in photos. (b) Measured current versus applied voltage with varying $\mathrm{k}$ in the perpendicularly aligned MWCNT-CY array device, with inset showing normalized resistance versus $k$ to extract a sensitivity value. (c) Current versus voltage with varying $k$ in the horizontally aligned MWCNT-CY array device. 
(a)

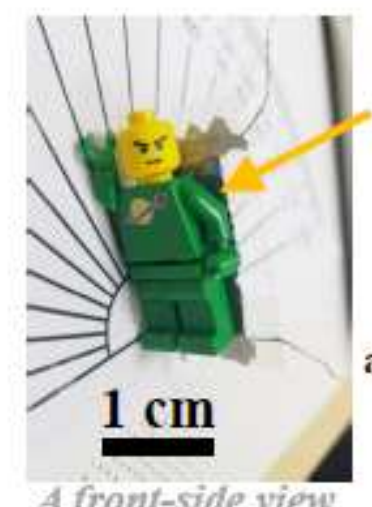

Bending sensor

attached

to the back of

a Lego figure

Perpendicularly

aligned MWCNT-CY

A front-side view

A back-side view

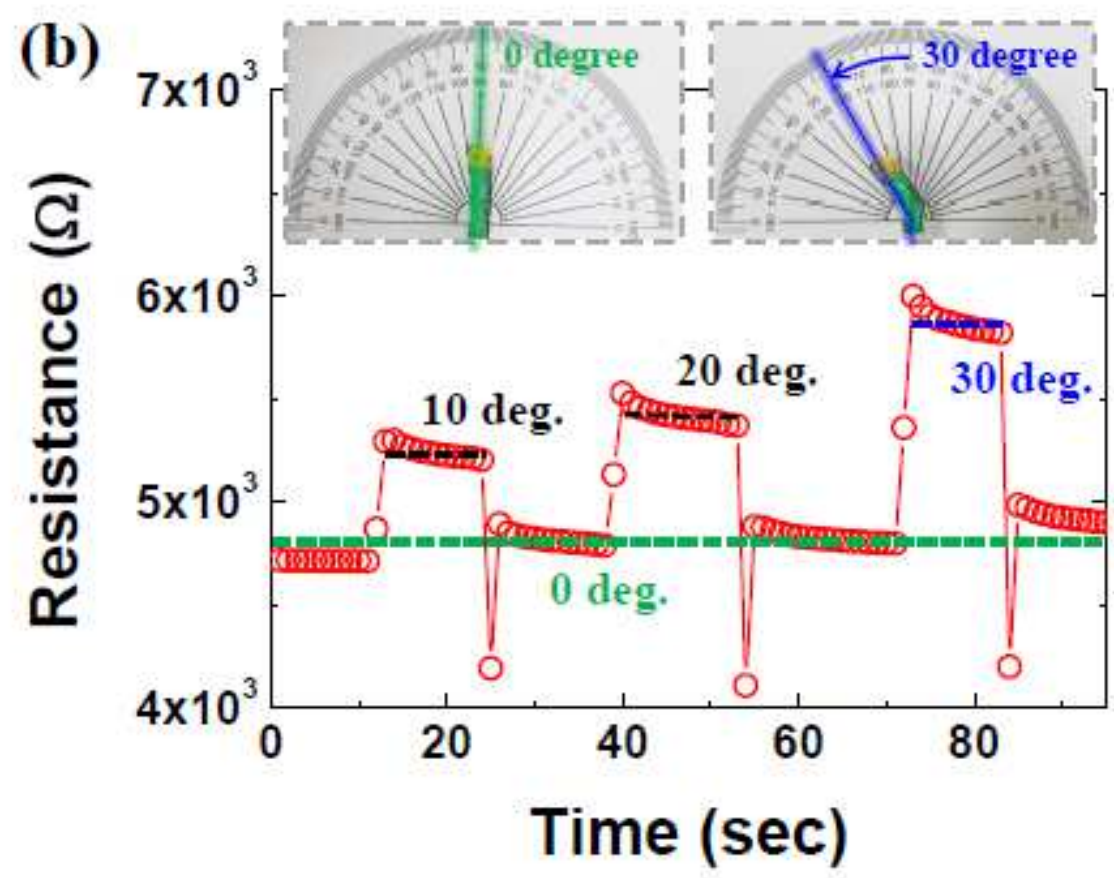

Figure 5

(a) Lego figure equipped with the bending sensor based on a perpendicularly aligned MWCNT-CY array as an active sensing material. (b) Measured resistance of bending sensor with varying degree of Lego figure bending. 

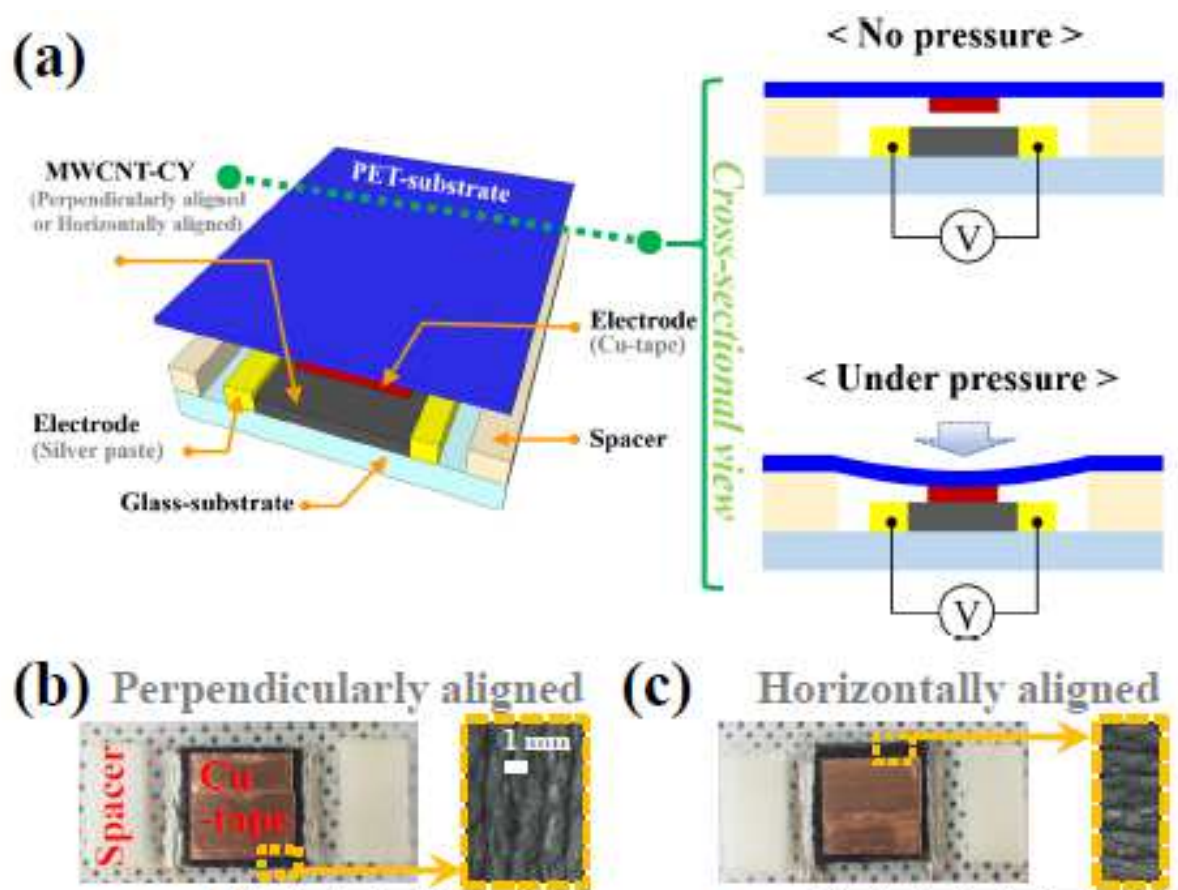

(c) Horizontally aligned
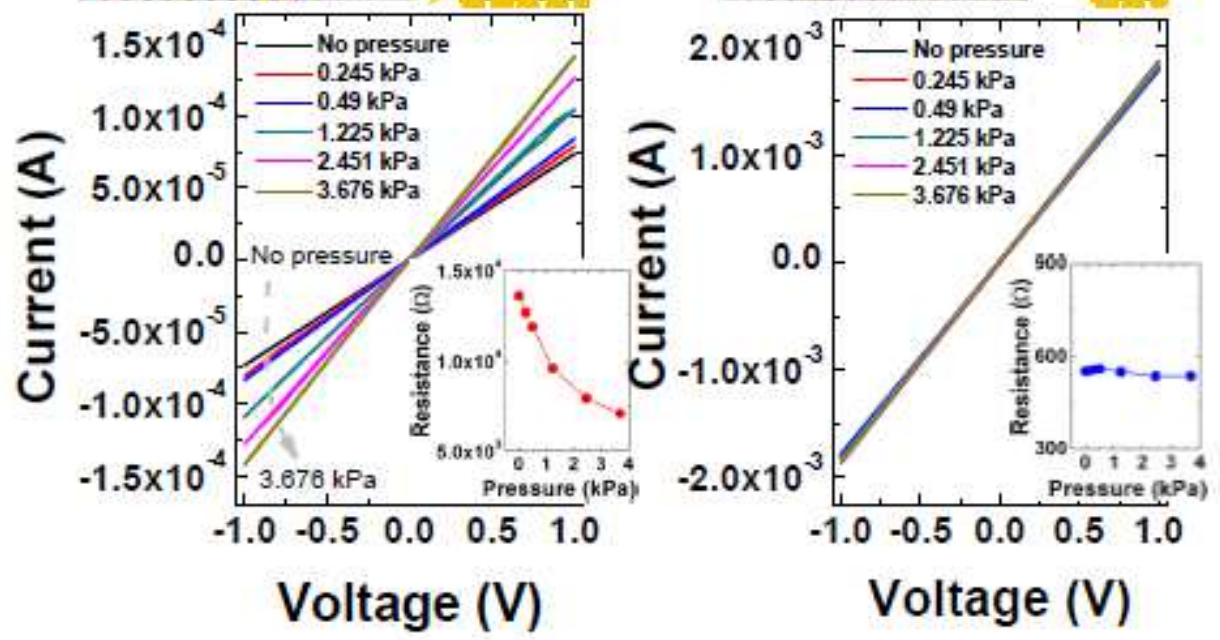

Figure 6

(a) Schematic architecture of device structure of a force sensor based on a MWCNT-CY array. Measured current versus applied voltage with varying pressure in the force sensor with (b) a perpendicularly aligned MWCNT-CY array, or (c) a horizontally aligned MWCNT-CY array. Plots in the insets show resistance variation versus pressure. 


\section{Force sensor}

with perpendicularly aligned MWCNT-CY

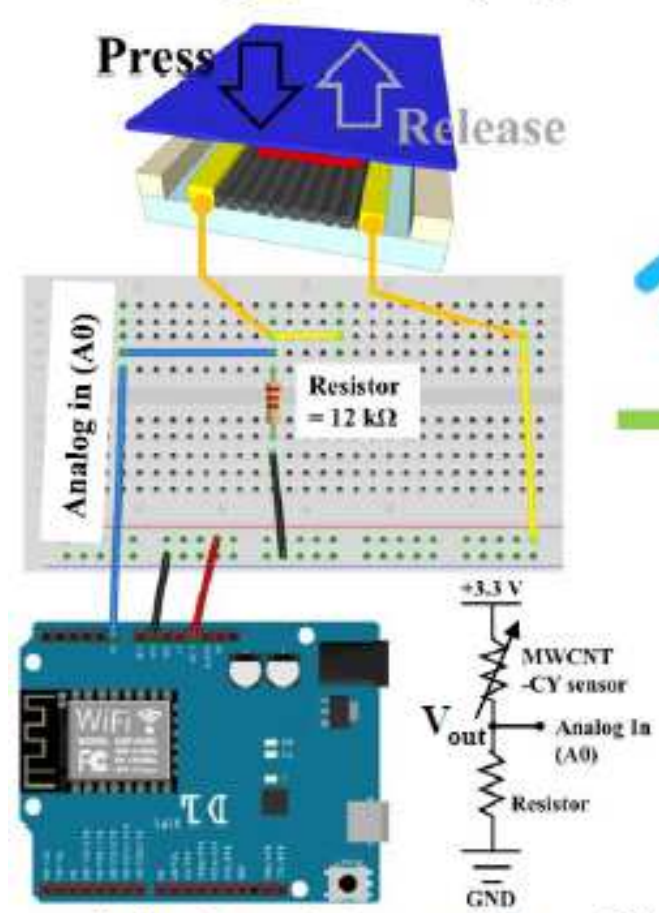

Arduino board (Wemos D1 wifi)

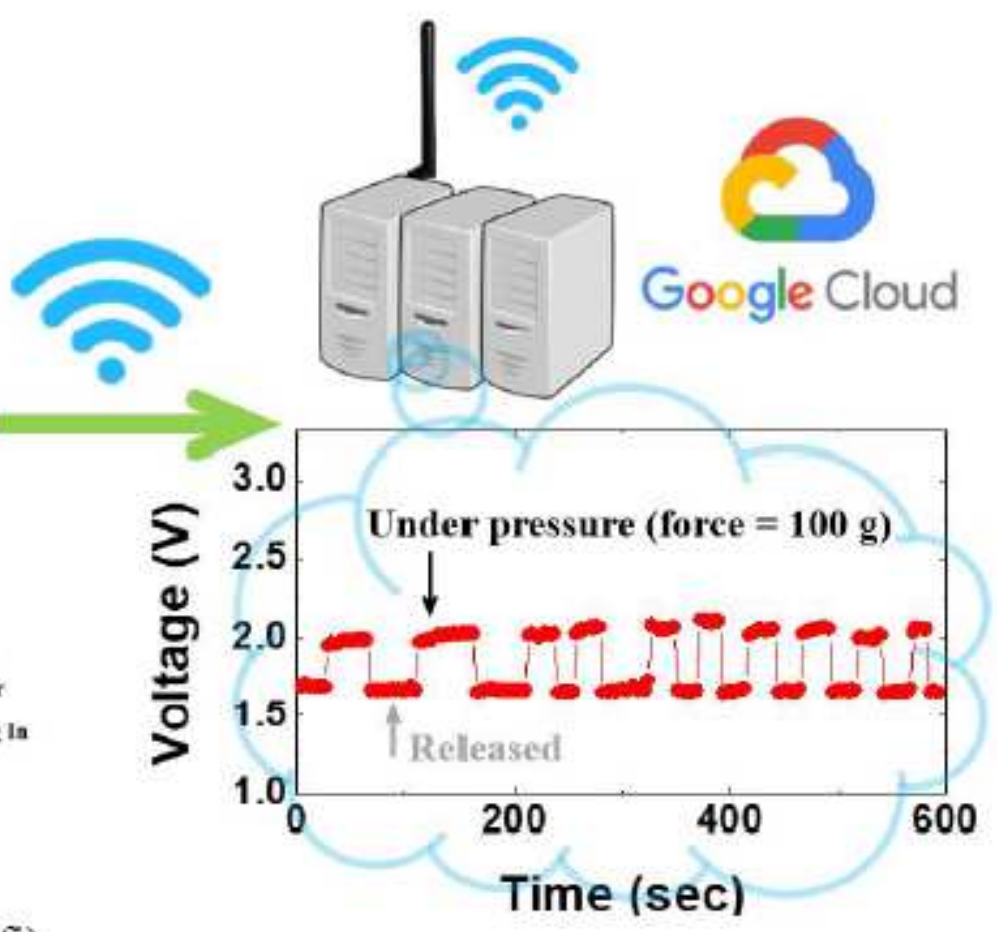

\section{Figure 7}

Simple system consisting of a force sensor, Arduino microcontroller, and real-time data storage in Google Cloud platform. The force sensor was based on a perpendicularly aligned MWCNT-CY array, and the plot shows the sensing data (automatically stored in Google Sheets) generated under repeated pressure and release operation. 


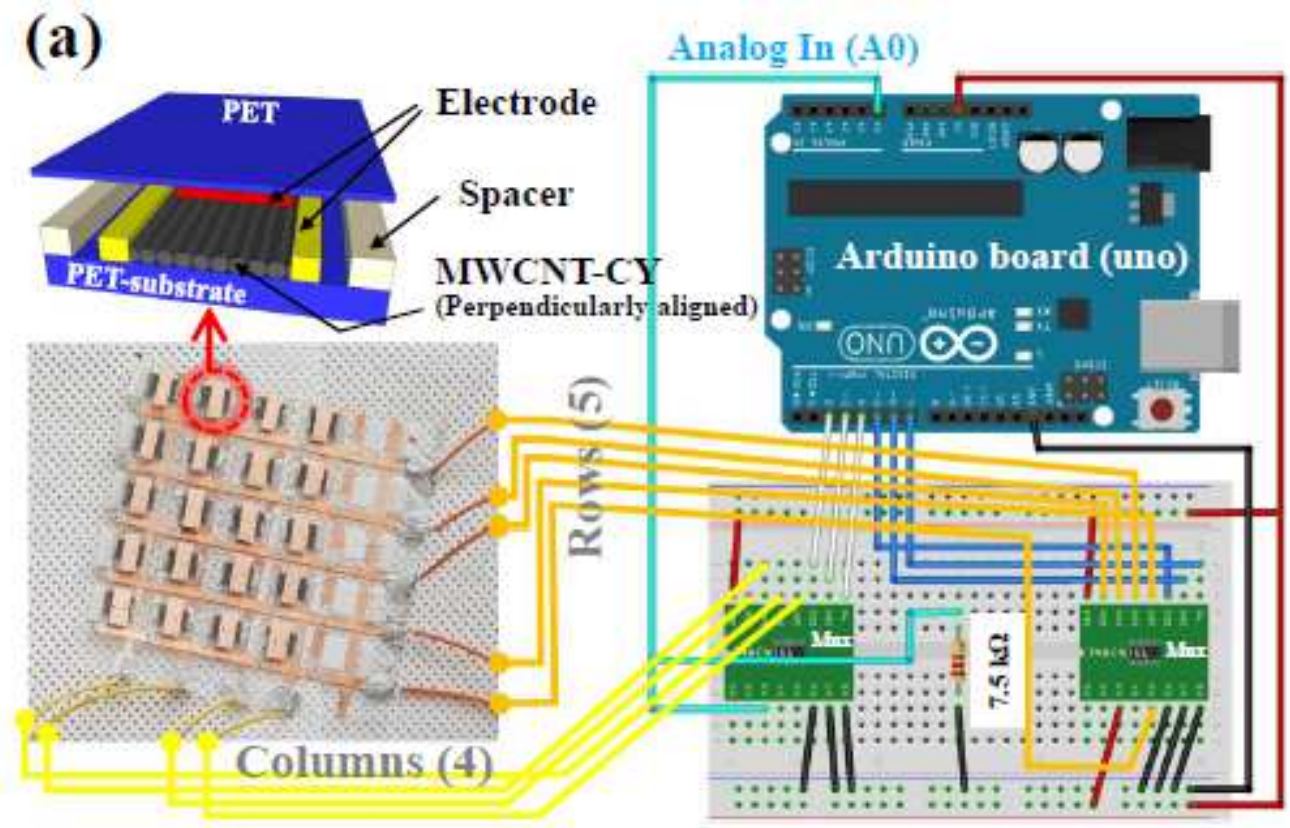

(b)
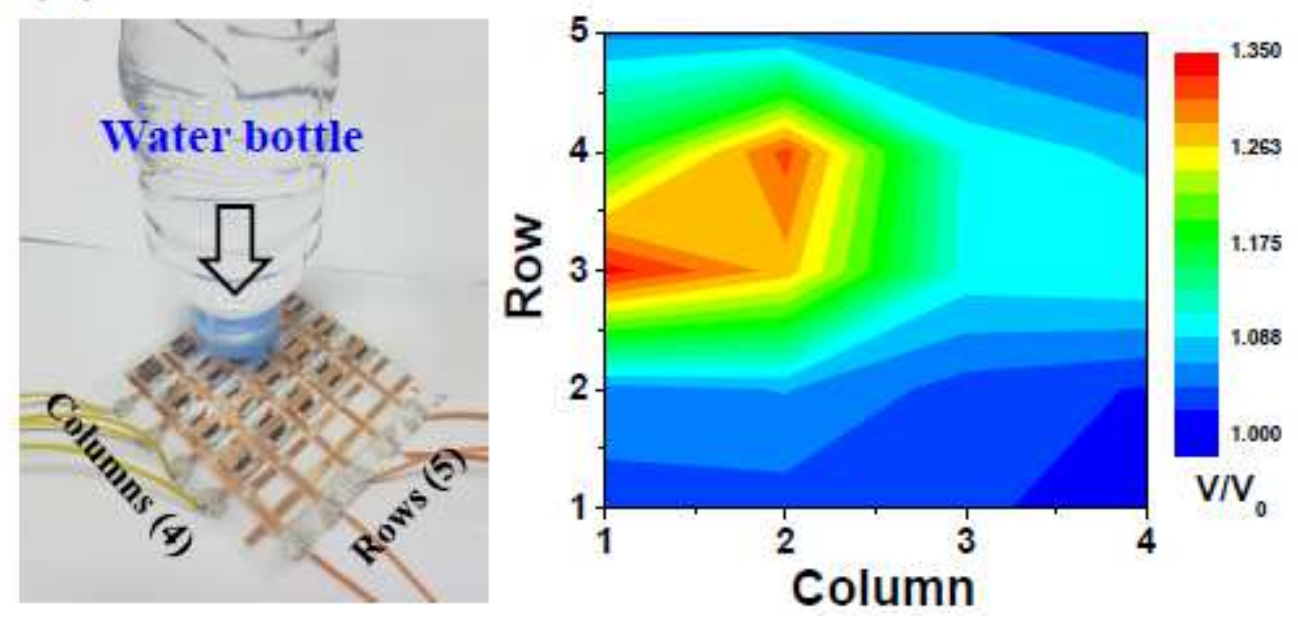

Figure 8

(a) System structure and detail electrical connections for a force sensor matrix with $5 \times 4$ pixels. (b) Photo and contour map (V/V0) of a force sensor matrix with the weight of a water bottle.

\section{Supplementary Files}

This is a list of supplementary files associated with this preprint. Click to download.

- Jeonscisupplfvre.pdf 